\title{
Technologies for safe and resilient earthmoving operations: A systematic literature review
}

\author{
S. Neda Naghshbandi ${ }^{*}$, Liz Varga ${ }^{a}$, Yukun Hu ${ }^{a}$ \\ ${ }^{a}$ Department of Civil, Environmental and Geomatic Engineering, University College London, London, WC1E 6BT, UK.
}

\begin{abstract}
Resilience engineering relates to the ability of a system to anticipate, prepare, and respond to predicted and unpredicted disruptions. It necessitates the use of monitoring and object detection technologies to ensure system safety in excavation systems. Given the increased investment and speed of improvement in technologies, it is necessary to review the types of technology available and how they contribute to excavation system safety. A systematic literature review was conducted which identified and classified the existing monitoring and object detection technologies, and introduced essential enablers for reliable and effective monitoring and object detection systems including: 1) the application of multisensory and data fusion approaches, and 2) system-level application of technologies. This study also identified the developed functionalities for accident anticipation, prevention and response to safety hazards during excavation, as well as those that facilitate learning in the system. The existing research gaps and future direction of research have been discussed.
\end{abstract}

Keywords

Technology innovation; object detection; earthmoving equipment; excavation; system safety; resilience engineering

\section{Introduction}

An excavation site is a complex system with a highly dynamic and transitory nature, and a unique and unstructured environment like any other construction site. The Lancaster University Computerized Intelligent Excavator (LUCIE) development has shown that when systems need to interact with unstructured and unpredictable environments, basic functionality becomes more complex and sensitive to environment conditions [1]. For example, the operation of earthmoving equipment in the complex and dynamic nature of an excavation site creates many safety risks which result in hazardous working situations that can cause accidental damage such as workers' injury or death, equipment damage or property loss [2]. In fact, contact with earthmoving equipment is either the primary or secondary cause of fatalities on construction sites [3][4]. In many construction projects such as road, dam, or building construction, intensive earthmoving equipment operations are required for excavating the site, and earthmoving operation costs account for more than $20 \%$ of total costs [5]. So accidents and disruption during excavation can create serious financial risks as well.

One of the major safety concerns during excavation is collision with other objects such as equipment, workers, structures, etc. [6][7][8]. Normally, the workspace of different items of equipment and the minimum distance among different objects in the excavation system are planned beforehand, but the dynamic and complex nature of the system imposes huge uncertainty on the planning phase, and makes it unreliable. The Occupational Safety and Health Administration (OSHA) of the United States has developed safety procedures and programs for excavation systems, but their adoption has not changed the rates of fatal and non-fatal injury, and construction firms still need to look for innovative strategies to enhance the safety of excavation systems [9], and eliminate collisions caused by earthmoving equipment. In addition, unforeseen damage to buried underground utilities during excavation is an important root of hazardous accidents and disruption in critical public services such as electric power, water supply, telecommunications, and air traffic control operations [10]. Although there are some standard guidelines for underground utility data collection and categorizing the quality of available utility data to facilitate risk assessment before excavation, accidents still occur from time to time, causing direct and indirect costs as well as severe injuries and loss of lives [11]. On the other hand, safety is becoming more important as the construction industry is going through a transition toward the smart construction site with fully automated and autonomous

\footnotetext{
* Corresponding author: S. Neda Naghshbandi (seyedeh.naghshbandi.18@ucl.ac.uk)
} 
equipment. Automated excavation has been reported since 1996 and the field continues to attract the interest of researchers in connected autonomous plant. Although robotics and the automation of repetitive tasks have been developed to specifically improve the excavation process, efficiency and safety, they have created new safety concerns for researchers, as well as technology developers and users. The question is how these technology innovations can take a proactive approach for handling safety risks and ensuring safety throughout the lifecycle of a system without human intervention. If autonomous mobile equipment such as intelligent excavators is going to become a commercial reality, these issues must be solved [12].

The normative safety management, also called "Safety I", that puts safety controls into effect through rules and standards has not been effective for the complex excavation environment [2]. And so far, the abovementioned problems remained unsolved. Because normative safety management only applies quantitative risk assessment, this is not sufficient for highly complex safety-critical "IT+human agent-based" systems such as automated excavation systems [13]. However, resilience engineering science, also called "Safety II", has emerged as a complementary approach to "Safety I" and enables excavation systems to respond to the limitations of normative safety management. But how could this help to eliminate collisions in excavation systems (automated or even non-automated systems)? Resilience engineering is a different prospect from normative safety management, as it focuses on the cognitive ability to anticipate and adapt in the case of accidents and failures (a focus on adaptive capacity), rather than a normative analysis of risk and security [14]. Here we review a relevant definition of resilience, to be able to show its importance and its effects on the excavation system. Also, it is necessary to define the concept of resilience to be able to differentiate it from normative safety management. Carmeli et al. [15] (p. 148) described resilience as a "proactive approach to safety management that recognizes the complexity and everchanging environment". Accordingly, health and safety management in the context of resilience engineering is achieved by applying prevention strategies for safety risks such as accidents that are able to deal with complex and dynamic systems [16]. In fact, resilience engineering refers to the adaptive capacity of the systems to cope with emerging and unknown risks to ensure system safety in a complex modern socio-technical system that is inherently risky [13]. Meanwhile, Francis and Bekera [2] (p. 101) defined system safety as "the application of engineering and management principles, criteria and techniques to achieve acceptable mishap risk, within the constraints of operational effectiveness and suitability, time and cost, throughout all phases of the system life cycle". It should be noted that the concept of system safety is more comprehensive than normative safety management, as it protects both the safety and productivity of the system, since safety risks disturb systems' functionality and productivity as well [16]. Therefore, resilience engineering and system safety science only support prevention strategies that can deal with unexpected system variations and permit the avoidance and anticipation of safety risks and accidents. This allows the systems to maintain a certain level of functionality and productivity all the time, even in the case of disruption. In other words, resilience engineering encourages project managers to learn, adapt and achieve success to create a safe environment in which people and the system are able to function safely [13], while caring for system performance [2].

As the resilience engineering approach relies on the adaptability of systems to cope with emerging and unknown risks, excavation systems should have the cognitive ability to anticipate and avoid different kinds of accidents. This necessitates the application of automated safety monitoring and object detection technologies, in order to add a cognitive ability to the system and facilitate the successful prevention of situations that could have serious safety consequences [7]. Furthermore, researchers have also conveyed the necessity of using real-time tracking technologies to enhance earthwork equipment safety and prevent accidents on construction sites [12]. Numerous studies have been conducted to develop and test different technology innovations to monitor, locate, and track different objects in the excavation system. This study aims to identify technologies that provide a timely and appropriate reaction and decision making support in unpredicted unsafe situations during operations. In this regard, a systematic literature review (SLR) has been conducted to address the following review question:

What technology innovations (including devices, methods, algorithms, etc.) are available to enhance system safety by anticipating and preventing accidents during excavation?

The review question is framed to explore the intersection of four main research areas, including technology innovations, safety, resilience, and the excavation system. Figure 1 shows the position of the study in these research areas, as well as the academic domains within the scope of the study. 


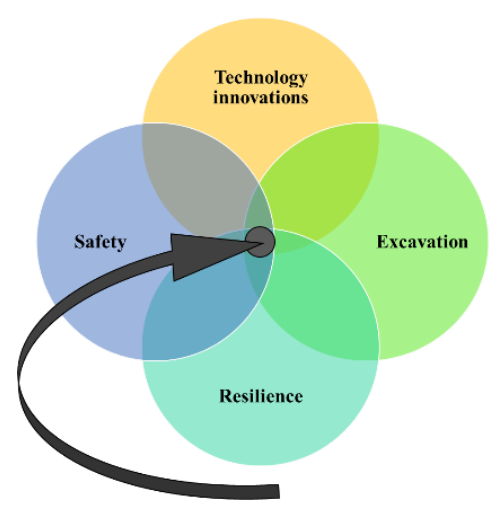

Fig. 1 Position of the review question in the area of the research.

The review helps to assess the capability of technologies to enhance operational system resilience engineering. The significance of this study is that it is inclusive of all the monitoring and object detection technologies and methods that have been used for enhancing the safety of excavation sites. Most of the other studies just discussed one particular technology application in construction site safety. This paper specifically addresses how different technologies have been used to improve excavation system safety. The findings are useful for earthmoving operation planners and managers to choose and integrate appropriate technology innovations that suit their operation system. This study also clarifies the level of technology readiness and further technology development requirements for achieving resilient excavation systems in which different smart and connected objects have the capability to avoid safety risks in unexpected and unsafe situations without human intervention. The findings are also useful for developing a 'technology roadmap' for smart, autonomous, and connected excavation. In fact, it demonstrates different research streams that are converging toward an ultimate aim beyond automation by taking essential small steps toward creating autonomous equipment that is necessarily connected to other construction plant on a site.

In the next sections, first the methodology for conducting the systematic literature review has been discussed in full detail to allow replication of the work by other researchers. Then the results have been presented in three subsections entitled 3.1 monitoring and object detection technologies, 3.2 underpinning methods and technologies, and 3.3 functionalities to increase adaptive capability. Subsection 3.1 presents different groups of technologies for monitoring the excavation system. Subsection 3.2 introduces two essential requirements for reliable and effective monitoring of excavation systems that can assure system safety. Subsection 3.3 talks about the capabilities driven by the available technology innovations to enhance excavation system safety. Section 4 discusses the contributions of the study to the body of knowledge, as well as research gaps and the future direction of study. Finally, the conclusion section summarizes the study.

\section{Methodology}

In order to map and evaluate the existing academic areas of knowledge, a literature review is often conducted, which helps to identify a research question to advance the current body of knowledge further [17]. Tranfield et al. [17] believed that systematic reviews boost the soundness and domination of the ensuing evidence and can lay out a certain basis for policy-makers and practitioners to make decisions. Accordingly, a comprehensive synthesis of the academic literature on technology innovations for safe and resilient excavation was organized adopting [17] a systematic review perspective. This approach was encouraged by systematic reviews in the field of medicine, and provides a clear explanation of the reviewer's procedures that will enable other researchers to reproduce and revise the literature review [18]. This review moved forward through the searching, screening, extraction and synthesis stages as follows.

\section{Searching}

Keywords and search terms were identified from the literature, consultation within the review team and several examinations. The selected keywords were then used to construct search strings with Boolean connectors "OR" to include appropriate synonyms. The reviewers determined the most appropriate search strings that matched the study. Then the search strategy was built by linking the strings with the Boolean operator "AND". The search strategy is illustrated in detail in the table below (Table 1) to ensure that the search can be duplicated. 
Table 1 Search strategy: combination of three search strings.

\begin{tabular}{lll}
\hline Theme & Search Strings & $\begin{array}{l}\text { Boolean } \\
\text { Operator }\end{array}$ \\
\hline $\begin{array}{l}\text { Technology } \\
\text { Innovation }\end{array}$ & $\begin{array}{l}\text { automat* OR technolog* OR autonom* OR intelligen* OR sensor* } \\
\text { OR "multi-sensor" OR gpr OR "ground penetrating radar" OR "data } \\
\text { fusion" OR human* }\end{array}$ & \\
$\begin{array}{l}\text { Resilience } \\
\text { Engineering }\end{array}$ & $\begin{array}{l}\text { reliab* OR resilien* OR robust* OR safety OR vulnerab* OR AND } \\
\text { "resilience engineering" OR "cognitive system engineering" }\end{array}$ & \\
\hline
\end{tabular}

Peer-reviewed journals were searched for relevant scientific literature. Four leading electronic databases, Scopus, Web of Science, EBSCO and PQ/ABI, were searched for articles whose titles and/or abstracts and/or keywords comprised no less than one of the search terms from all three strings. This search across all databases returned a total of 857 titles after deleting duplicated articles (see Table 2). The search was limited to TITLE-ABS-KEY and English articles in peer-reviewed journals. For subject area exclusion, see Table 3.

It should be noted that a scoping study of the field was conducted prior to the systematic literature review. The purpose of the scoping study was to specify where and how the proposed systematic review fits into the current body of knowledge. Exhaustive literature searches of published studies were conducted to ensure that the review results took into account all the available evidence and were based on the best quality contributions. Significant time was spent on constructing the search strings. The results of each search strategy were reviewed to determine if the results can answer the review question.

Table 2 Total number of results for the search strategy.

\begin{tabular}{llllll}
\hline Data Base & Scopus & Web of Science & PQ/ABI & EBSCOhost & $\begin{array}{l}\text { Total after deleting } \\
\text { duplications }\end{array}$ \\
\hline Number of results (articles) & 672 & 411 & 18 & 60 & 857 \\
\hline
\end{tabular}

Screening

The process of selecting studies had several stages. Initially, all possibly relevant articles were identified through reviewing the title and abstract, which led to 110 articles. The determinant studies met all the inclusion criteria and manifest none of the exclusion criteria incorporated into the review. These criteria are illustrated in Table 3. Relevant sources were restored for a precise evaluation of the full text and the determinant ones including 49 articles remained in the systematic review.

Table 3 Inclusion and exclusion criteria.

\begin{tabular}{ll}
\hline Criteria & Inclusion \\
\hline
\end{tabular}

Study type Review, experimental, conceptual, case study, analytical, and modelling

Conference papers studies. Peer-reviewed.

Language English

Sector Construction Industry, Construction Site, Construction Equipment, Earthmoving Equipment, Excavation, Civil Engineering Project, Underground Construction, Adjacent Structures, Coal Mine Roadway Excavation, Tunnelling Projects.
Other Languages

Medicine, Biochemistry, Genetics and Molecular Biology, Physics and Astronomy, Chemistry, Dentistry, Neuroscience, Chemical Engineering, Immunology and Microbiology, Pharmacology, Toxicology and Pharmaceutics, Nursing, Anthropology, Evolutionary Biology, Zoology, Paleontology, BiochemistryMolecular Biology, Legal 
Medicine, Forestry, Physics, Genetics Heredity, Dentistry Oral Surgery Medicine, Entomology, Life Sciences Biomedicine Other Topics, Neurosciences Neurology, Ophthalmology, Surgery, Mathematical Computational Biology, Oceanography, Paediatrics, Pharmacology, Pharmacy, Research Experimental Medicine, Toxicology, Cardiovascular System Cardiology, Developmental Biology, Endocrinology Metabolism, Fisheries, Gastroenterology Hepatology, History, Linguistics, Medical Informatics, Neural Network, Organic chemistry.

\section{Date $\quad 1996-2020$}

Relevance Sensors and Monitoring, Worker Safety, Equipment Safety, Utility Network Safety, Construction Site Safety, Robotic Excavator, Object Detection, and Tracking, Accident and Strike Avoidance, System Safety, Resilience Engineering. Only external threats, such as dynamic and static objectives in the path of excavation.
Various built-in sensing devices that could provide a wide range of data from the machine itself such as engine operating parameters to facilitate operating the equipment efficiently. Sensors that measure structural displacement in soil, as well as soil-equipment interactions/forces. Types of technologies to ensure the structural stability of the tunnel during excavation. Preoperation planning techniques to assign sufficient numbers of equipment and enough space and adequate barriers to avoid accidents. Normative safety management and related planning techniques. Techniques for data accuracy and visualization of uncertainty.

In order to review the best quality evidence, the remaining articles were reviewed based on the pre-determined quality criteria covering alignment between the research questions and articles' theoretical background, methodological approach, findings and contribution [18] (see Table 4 as an example). Each part scored from 0 to 3 , and the articles with an average score of 1.3 could remain in the study. But two cases with average scores of 1 and 1.25 remained, as the authors thought their small contribution still worth including in the results. The quality appraisal resulted in eliminating 4 more articles. In addition, 15 articles were added as a result of snowballing and expert suggestions, which made a total inclusion of 60 papers for the review. The results cover a broad range of journals, and the majority of the papers are from the Journal of Automation in Construction (22 out of 61 articles) (see Table 5), covering a range of disciplines taking a variety of research methodological approaches (see Table $3)$.

Table 4 Full text review quality criteria.

\begin{tabular}{llllll}
\hline Title & $\begin{array}{c}\text { Theoretical } \\
\text { Background }\end{array}$ & Methodology & Findings & Contribution & Average \\
\hline $\begin{array}{l}\text { Earthmoving equipment } \\
\text { automation: A review of technical } \\
\text { advances and future outlook }\end{array}$ & 1 & 1.5 & 1.5 & 1.5 & 1.375 \\
$\begin{array}{l}\text { Enhancing coordination and safety } \\
\text { of earthwork equipment operations } \\
\text { using multi-agent system }\end{array}$ & 3 & 3 & 2.5 & 3 & 2.875 \\
$\begin{array}{l}\text { Computer-aided DSS for safety } \\
\text { monitoring of geotechnical } \\
\text { construction }\end{array}$ & 2.5 & 2.5 & 2.5 & 2 & 2.375 \\
\hline
\end{tabular}

Publications were assessed in terms of the relevance of their contribution to our review and we avoided subjectivity through close scrutiny and informal inter-rater assessment. The authors are very confident that no 
relevant paper was eliminated based on critical appraisal. The synthesis process validates the relevance in terms of each included study's contribution by reference to extant theory. We assessed quality according to several domains including quality of reporting, methodological rigour and conceptual depth and breadth. Table 5, which is adapted from [19], is added to define the methodological, conceptual, and reporting quality dimensions that we considered during the review.

Table 5 Three dimensions of quality for critical appraisal. Adapted from [19].

\section{Quality Dimension}

\begin{tabular}{|c|c|c|c|}
\hline Features & Methodological & Conceptual & Reporting \\
\hline Definition & $\begin{array}{l}\text { Extent to which a study's } \\
\text { design, conduct, and } \\
\text { analysis minimized } \\
\text { selection, measurement, } \\
\text { and confounding biases }\end{array}$ & $\begin{array}{l}\text { Extent to which a concept } \\
\text { is clearly articulated to } \\
\text { facilitate theoretical } \\
\text { insight }\end{array}$ & $\begin{array}{l}\text { Extent to which a paper } \\
\text { provides information about } \\
\text { the design, conduct and } \\
\text { analysis of a study }\end{array}$ \\
\hline Constructs & Trustworthiness & Insightfulness & $\begin{array}{l}\text { Accuracy, completeness, } \\
\text { transparency }\end{array}$ \\
\hline \multirow[b]{2}{*}{$\begin{array}{l}\text { Component example of } \\
\text { criteria }\end{array}$} & Study & Study & Research paper \\
\hline & $\begin{array}{l}\text { Were the statistical tests } \\
\text { used to assess the main } \\
\text { outcomes appropriate? }\end{array}$ & $\begin{array}{l}\text { Are there clear translatable } \\
\text { concepts? }\end{array}$ & $\begin{array}{l}\text { Is the hypothesis/aim/ } \\
\text { objective of the study clearly } \\
\text { described? }\end{array}$ \\
\hline
\end{tabular}

Table 6 Reviewing journals.

\begin{tabular}{llll}
\hline Journal & $\begin{array}{l}\text { Number of } \\
\text { Papers }\end{array}$ & Journal & $\begin{array}{l}\text { Number } \\
\text { of Papers }\end{array}$ \\
\hline Advanced Engineering Informatics & 4 & Journal of Computing in Civil Engineering & 5 \\
Automation in Construction & 22 & $\begin{array}{l}\text { Journal of Construction Engineering \& } \\
\text { Management }\end{array}$ & 5 \\
Autonomous Robots & 2 & Knowledge-Based Systems & 1 \\
$\begin{array}{l}\text { Computing and Control Engineering } \\
\text { Journal }\end{array}$ & 1 & Reliability Engineering and System Safety & 3 \\
$\begin{array}{l}\text { IEEE Sensors Journal } \\
\text { International Journal of Advanced }\end{array}$ & 1 & Sustainability (Switzerland) \\
Manufacturing Technology & 1 & Visualization in Engineering & 1 \\
$\begin{array}{l}\text { International Journal of Advanced Robotic } \\
\text { Systems }\end{array}$ & 1 & Sensors (Switzerland) & 1 \\
$\begin{array}{l}\text { International Journal of Distributed Sensor } \\
\text { Networks }\end{array}$ & 1 & Safety Science & 2 \\
Journal of Applied Geophysics & 1 & Journal of Aerospace Engineering & 1 \\
Journal of Coal Science and Engineering & 1 & Geotechnical Engineering & 1
\end{tabular}




\section{Extraction and synthesis}

Data-extraction forms were employed to reduce human error and bias, and these contained general information such as the title, abstract, authors, publication details as well as specific information and notes on emerging themes. The full list was: No., Reading Date, ABS Rating, Theme, Title, Author, Journal, Year, Ref. Number, Study Type, Industry, Method, Research Questions/Objectives, Theoretical Base, Methodology, Data Source, Findings, Strengths, Weakness/Limitation, Further Research, Additional Notes, Quality Appraisal (Theoretical Background, Methodology, Findings, Contribution). The acquired information was synthesized by summarizing, integrating and cumulating the extracted data. Template analysis was used to code the selected articles.

King (2012), cited in [20], defined template analysis as "a style of thematic analysis of textual data that allows a flexibility of coding structure through the use of tentative a priori or initial codes, which allow researchers to focus on finding relevant information from the text". The initial codes tried to capture the core focus of the paper and the paper methodology. For example, [21]was coded as equipment-related collision prevention, location-based guidance system, system level, multi-agent system. Equipment-related collision prevention was the main focus of the paper, a location-based guidance system was the kind of technology/sensors that were applied in the study, the system level was the scale on which the technology had been used, and finally the multi-agent system was the paper's methodology. These initial codes can be advanced by adding, deleting, or merging when further themes appear in the text [20]. Second-order coding followed the first-order coding and grouped the earlier codes into categories and higher-level themes. The authors used colour-coding in this stage, where the grouped articles appeared in the same colour, and the colours of approximately similar groups were in close ranges. Ultimately, the "final" template was analysed and interpreted, categorized in three main areas that are discussed in detail in the next section.

\section{Results}

To meaningfully demonstrate the identified technology innovations and their impacts on excavation system safety, the findings have been categorized and presented in three sections: (1) monitoring and object detection technologies; which presents available technologies for monitoring the excavation system and detecting different objects at the excavation site, (2) underpinning methods and technologies; which discusses the required methods and technologies that underpin a reliable and effective monitoring and object detection system, (3) anticipation, prevention, response and learning methods; which introduces the application of monitoring and object detection technologies for developing fundamental functions that support accident prevention, anticipation as well as the response to potential accidents in the excavation system. Figure 2 presents these different categories of the technologies and methods that have been developed to increase excavation system safety. 
Technologies for safe and resilient excavation systems

Above-Ground monitoring and object detection technologies

Below-Ground object detection technologies

The underpinning technologies and methods that enable both above and below ground technologies

\begin{tabular}{|c|c|c|c|}
\hline \multicolumn{2}{|c|}{$\begin{array}{c}\text { Multisensory approaches and data fusion for } \\
\text { increasing data accuracy and reducing } \\
\text { uncertainties }\end{array}$} & \multicolumn{2}{|c|}{$\begin{array}{l}\text { Communication, and information technologies } \\
\text { to facilitate system-level application of } \\
\text { technology innovations }\end{array}$} \\
\hline \multicolumn{4}{|c|}{ Functionalities to increase adaptive capability } \\
\hline $\begin{array}{l}\text { Anticipation: } \\
\text { proximity warning }\end{array}$ & $\begin{array}{l}\text { Prevention: accident- } \\
\text { free navigation }\end{array}$ & $\begin{array}{c}\text { Response: } \\
\text { knowledge-based } \\
\text { adaptive control } \\
\text { systems }\end{array}$ & Learning: AI and VR \\
\hline
\end{tabular}

Fig. 2. Different types of technology available to enhance excavation system safety.

\subsection{Monitoring and object detection technologies}

The reviewed literature showed that various technologies are required to comprehensively monitor and detect all the different objects involved in the excavation system, such as construction equipment, workers, and the utility network. These technologies can be categorized as two main groups: 1) above-ground monitoring and object detection technologies and methods; technologies and methods to monitor and detect construction equipment, workers, and the utility network above the ground; 2) below-ground utility detection technologies and methods; technologies and methods to detect the utility network below the ground at the construction site. Categorizing these technologies and methods in different groups clarified the vital role of each group in the overall system safety as well as the different technology level readiness of each group. Therefore, the research gap and areas for further research and advancement to achieve safer and resilient excavation systems in each group could be underlined.

\subsubsection{Above-ground monitoring and object detection technologies and methods}

There are some technologies, devices and sensors available that are capable of monitoring the excavation system and detecting above-ground objects in the vicinity of the excavator. The two main forms of accidents that can occur above the ground during excavation are excavator to equipment collisions, and excavator to worker collisions, which can be anticipated and prevented by utilizing automatic monitoring and object detection systems. Figure 3 presents and categorizes the identified technologies and methods for anticipating and preventing accidents involving above-ground objects during excavation. 


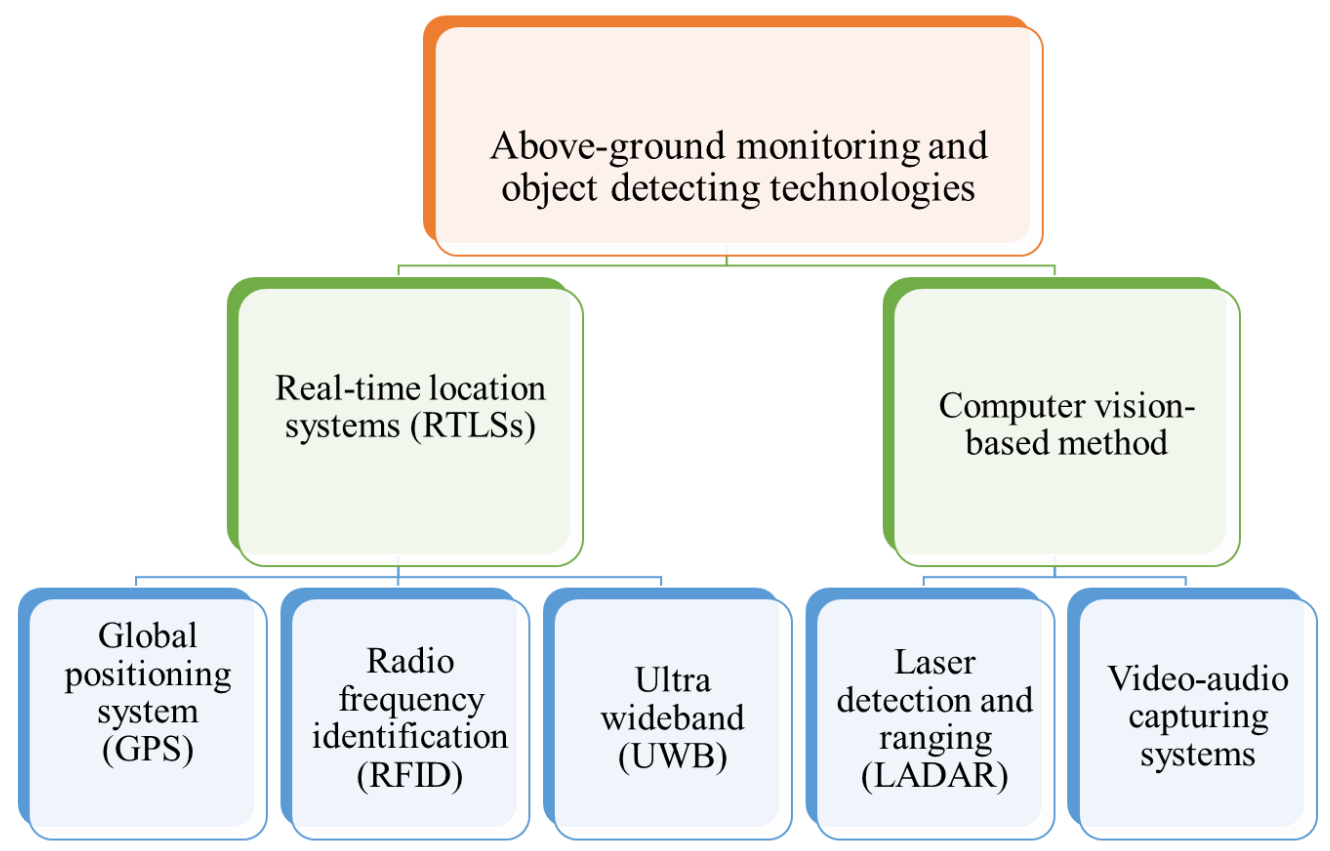

Fig. 3. Hierarchy of technologies and methods for monitoring and detecting above-ground objects during excavation.

\subsubsection{Real-time location systems (RTLSs)}

Construction sites utilize novel automated data capturing technologies for object detection above the ground, such as the Global Positioning System (GPS), Radio Frequency Identification (RFID), and Ultra-Wideband (UWB), which are called real-time location systems (RTLSs), to overcome the deficiencies of traditional monitoring methods [3].

Real-time positioning devices such as GPS and UWB are used as automated vehicle tracking technologies to provide 3D data on the location of the equipment and stationary plants such as excavators, which are valuable data for planning and safety management of resources, as well as accident prediction and prevention on construction sites. However, such data cannot distinguish whether the equipment is idle or active - so-called pose and state identification - while pose and state data can increase the automated monitoring system's functionality [22][3]. The pose is the most essential information required for monitoring of the equipment's operation. The pose of the equipment can be effectively used to detect hazardous conditions and identify the working state (idle, active, etc.) of the equipment [22][3]. The equipment pose is a spatial aspect that represents the equipment's location and orientation at a certain time. Three RTLS data collectors positioned on the excavator's upper structure or one RTLS data collector and a digital gyroscope can establish the orientation reference frame for the excavator [3].

Another of the most crucial requirements for equipment safety evaluation is capturing near real-time data rather than real-time data [4]. Real-time data is defined as "pertaining to the performance of a computation during the actual time that the related physical process occurs, in order that results of the computation can be used in guiding the physical process", and near real-time data is defined as "pertaining to a delay introduced, by automated data processing, between the occurrence of an event and the use of the processed data", according to Federal Standard 1037C [4] (p. 12). A real-time scanning device requires intensive computational effort for site scanning. Updated 3D models and accurate equipment pose data integration can eliminate the need for real-time scanning by every equipment [21]. Many technologies are available that can capture the equipment pose data, such as laser-based methods, an inertial measurement unit (IMU), and active and passive marker-based motion tracking systems [4].

In addition, location-based guidance systems (LGSs) are produced by RTLS geo-positioning data, 3D design models, and digital terrain model (DTM) integration to support construction equipment operators. This support can be a cabin-mounted display providing visual guidance to operators, or control of the equipment movement and position [21]. Automated machine control and guidance (AMC/G) is provided by accessible LGSs in the market, which adopt expensive high-accuracy GPS [21]. Other cost-effective systems that utilize RTLSs such as UWB are also proposed [21]. Several systems such as the Connected Site and SiteLINK 3D are under development 
to provide telematics information to and from a central office and other equipment for the AMC/G system. The manufacturers that offer AMC/G solutions include Caterpillar's MineStar, Leica's Jigsaw, Topcon, and Trimble. These solutions provide different levels of capabilities, from simple operator guidance to fully autonomous machine control. An ontology-based information management system data communication standard was developed based upon the existing AMC/G infrastructure by [23].

\subsubsection{Computer vision-based method}

The recognition of construction operational resources has an important role in achieving fully automated construction [24]. Hence, computer vision-based (CV-based) methods have been significantly used to automatically monitor and detect people, buildings, plant, materials and equipment on construction sites from images or videos to improve safety and productivity [25] [26]. Conventional surveillance cameras have not been efficiently used as a result of the labour-intensive process of manually extracting data from the images and videos before they are eliminated to save memory [22]. Therefore, high-resolution digital wireless cameras and highcapacity storage devices have been adopted to a great extent at construction sites as a result of their good enough return on investment, as well as producing helpful management information for construction engineers/managers to monitor and control sites remotely and dynamically [24]. Normal cameras, as a cheap alternative, produce only two-dimensional (2D) projections of the real world, which reduces the analysis to 2D. Other sensing devices can be engaged with video cameras to collect more data from the scene [27]. And three-dimensional (3D) reconstruction techniques can recover the geometric relationships among $2 \mathrm{D}$ images to automate quality checking and progress monitoring [26]. Alternatively, 3D coordinates can be produced by video/audio capturing systems such as laser detection and ranging (LADAR), infrared cameras, or multiple cameras for the same scene, but these are expensive and must remain accurately calibrated to work perfectly [27]. Azar and McCabe [28] developed a framework to extract a dump truck's pose data by static image processing and video analysis. They claimed that the framework serves for the real-time estimation of productivity, calculation of service/idle time, and detection of potential collisions.

The recent growth of CV-based monitoring systems is not limited to research and academia. Service providers are delivering fully automated productivity estimation systems using CV-based methods. For instance, indus.ai, a Canadian service provider, is offering CV-based performance analytics system for construction sites [4]. Despite the broad adoption of the method, it has some limitations for construction site application. For example, only a few categories of objects in natural scenes have been approved by many object recognition algorithms which have been developed for this method [24]. Furthermore, most studies in this area are focused on using CV based methods for detecting and locating the construction equipment as a single object, regardless of its parts [4].

However, the review showed the developments that artificial intelligence (AI) has allowed in utilizing computer vision-based methods for safety enhancement of excavation sites. The deep learning method can be applied to automatically obtain specific features at high levels of abstraction [29]. This method also facilitates learning from data with various levels of representations. Image features can be automatically obtained via integrating computer vison with learning methods or can be used for learning from training data [29]. One popular type of deep learning method is the use of Convolutional Neural Networks (CNNs), which is more accurate compared to the deep neural networks method as it assigns robust and effective classifiers [30]. Specific applications of deep learning for detecting objects at excavation sites have been addressed in the following studies.

Tajeen and Zhu [24] classified trends in computer vision-based methods' object recognition into three main categories: (1) geometry-based, (2) appearance-based, and (3) feature-based methods. Some of these are explained in more detail below.

Histogram of oriented gradients (HOG) is a robust algorithm to detect rigid objects. In this algorithm, the computed gradients of the grey-scaled image are discretized into spatial and orientation cells to form a descriptor vector. Then a single classifying vector is generated by the positive and negative vectors. This detector uses a sliding window technique to check all locations and scales of an image. If the result exceeds a determined threshold, that window is accepted as a target [22]. Objects that change shape are definitely more problematic to detect and some research has focused on human detection and pose identification, seeing the complicated configurations of the human body [22]. Many of the developed models have used part-based and pictorial structures to find a group of parts of a semantic object arranged in a deformable configuration. One of the forefront algorithms is the latent support vector machine (Latent SVM) part-based model [22]. This model uses a modified HOG detector, termed a root filter, to detect apparent candidates for the object within the image. It then searches for the parts of the object at twice the spatial resolution relative to the features grabbed by the root filter inside the 
captured root areas [22]. Azar and McCabe [22] developed a part-based object recognition model that employed discriminately trained HOG classifiers to detect hydraulic excavators in different poses. This method first scans the image for the first part of the boom and then searches for the second part in potential configurations around the boom to confirm the detection and appraise the pose of the machine. This algorithm has almost the same performance as general HOG classifiers, but it noticeably surpasses the HOG detectors in higher thresholds, where it showed higher detection rates with fewer false alarms.

Kim et al. [26] proposed a deep convolutional network-based object-detection method to obtain the appropriate accuracy to robustly recognize objects such as construction equipment. Generally, a deep convolutional network can achieve high performance in various visual tasks, but in construction sites it is not easy to use as there is not enough publicly available data for training. So transfer learning was used, which transfers a large amount of training data - the knowledge of models trained in other domains - to the construction industry. A benchmark data set was also created for five classes of various shapes and poses: loader, dump truck, excavator, concrete mixer truck, and road roller, to figure out the generalization performance of the proposed model. The author claimed that the proposed model can be used on construction operations for context interpretation in order to produce productivity and safety managerial information [26].

Soltani, Zhu and Hammad [31] tried different ways to enhance the current CV-based method performance including: (1) the application of two regular surveillance cameras as a stereo vision system on a large construction site, and developing a multiple cameras calibration guideline using sensitivity analysis; (2) data fusion of RTLS and video images, which results in a reduction of the processing effort for detecting excavator parts, at the same time raising the detection accuracy by narrowing the search scope for the detectors; (3) elaborating a previous clustering technique (k-means) and skeleton extraction algorithms; (4) estimation of the 3D pose of the excavator from 2D skeletons of the excavator, taking each camera's views; and finally (5) testing the skeleton extraction and 3D pose estimation methods in a case study that verified the applicability of the suggested methods [31]. In addition, Fang et al. [25] utilized an IFaster R-CNN to detect workers and excavators in real time on construction sites in order to produce information for managers to optimize safety and planning decisions (Fig. 4). A database of objects from construction sites was created to train a CNN model to accurately detect their occurrence. It is an easy-to-use and low-cost solution to detect objects on-site in real time at a speed of $0.101 \mathrm{~s}$ per image. Automatic detection of unsafe actions (e.g., hardhat-wearing detection) and unsafe conditions (dangerous areas) during construction are the likely benefits of the method [25]. Figure 4b shows that small-scale objects can also be detected.
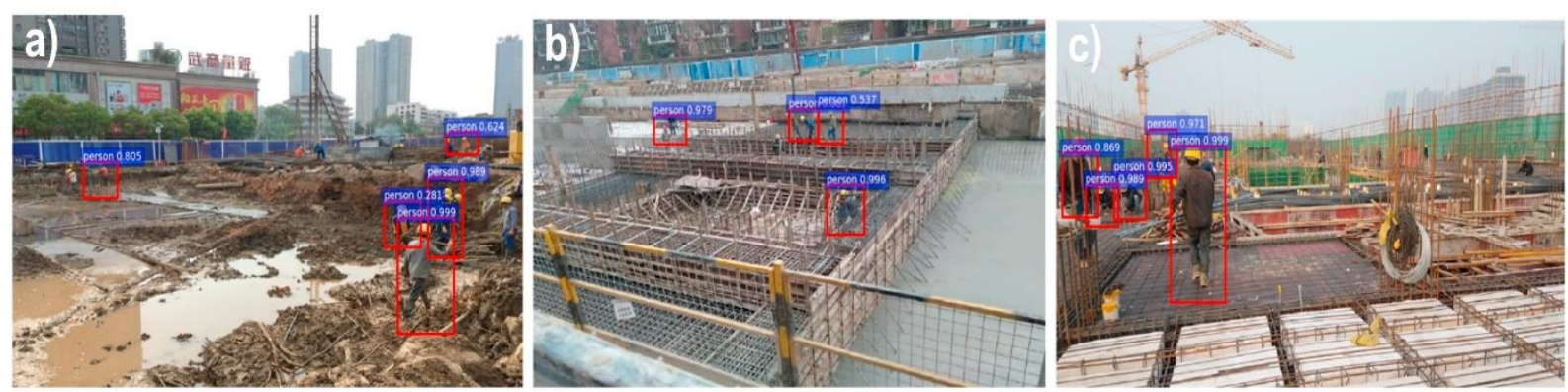

(a) Method of Faster R-CNN
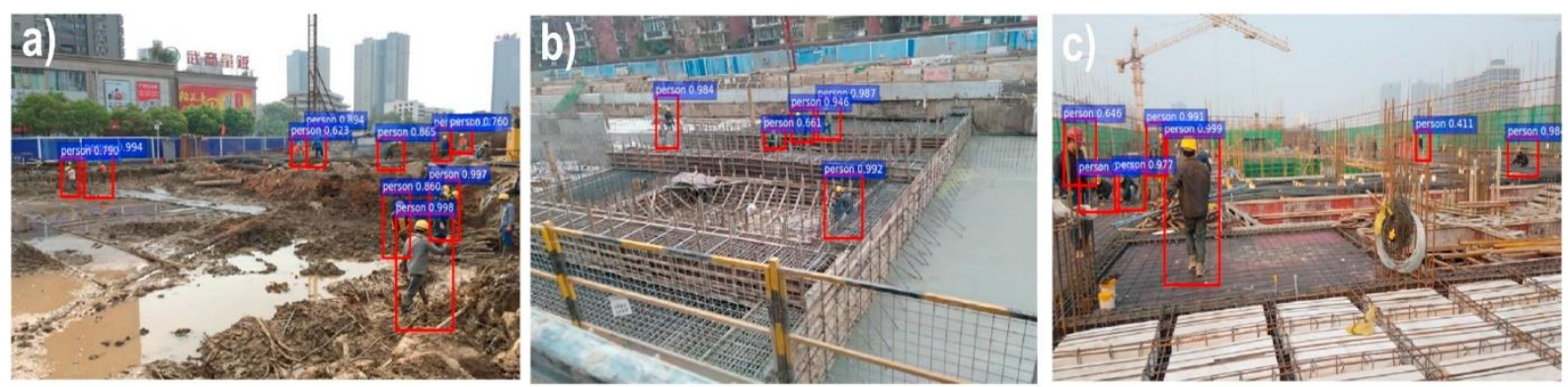

(b) Method of IFaster R-CNN

Fig. 4. Examples of detection results for Faster R-CNN and IFaster R-CNN. Adapted from [25]. 
In general, construction asset recognition and classification requires techniques such as digital data acquisition, feature description, and machine learning [32]. And 3D as-built data are preferred and more helpful in contrast to 2D data, subject to the additional spatial data and visualization flexibility. In fact, as-built sensing and visualization are the most auspicious technologies that are greatly expanding the utilization of site information. Chen, Zhang and Wang [32] introduced and compared three as-built 3D data-obtaining technologies including: a 3D range imaging camera, photogrammetry, and laser scanning, which can produce a 3D point cloud to support applications such as construction hazard recognition, highway asset management, etc.

Laser scanning (LS) can be used as a supplementary technology as it has proved to be an accurate tool for automatically producing point cloud models, as-built visualization, and comparison of as-built scenes, 3D mapping, reconstruction and geometry documentation of objects [33][34]. It also has the potential to precisely track activities during construction [33]. LS gathers an individual point's range ( $\mathrm{x}, \mathrm{y}$, and $\mathrm{z})$ and intensity in a scene. A laser scanner restores the data as a point cloud that can be visualized through various types of software. Laser scanners are capable of large range measurements at high resolution, millions of point locations can be delivered with high accuracy, and are mostly not restricted by climate conditions during operation, and it is also not affected by harsh field conditions [33][34]. These features allow for the construction of as-built states and 3D visualization in a virtual environment [34]. LS can also be used for specific tasks at an excavation stage, for example profile checks and cut volume calculations. More importantly, LS surveys can deal with asset inventory, which enables 3D models that have a place for complete imaging and positional information, and can be used for detailed inspection and for documentation [33]. Furthermore, these 3D models supply base data for planning renewal projects [33]. A scanning laser sensor can be used to detect objects within the vicinity of the excavator [12]. Marks et al. [35] developed an algorithm to evaluate blind spots of construction equipment by utilizing a laser scanner capable of creating a 3D spatial point cloud from both inside and outside the equipment's cabin. According to Marks et al. [35], the blind spot measurement data give practical insights on how construction equipment design influences the visibility of an equipment operator, and consequently safety problems that arise from proximity issues.

According to Chen, Zhang and Wang [32], very little examination of 3D range imaging cameras has been done in construction compared to their application in the virtual reality and gaming fields. Although they are portable and less expensive than laser scanners, they do not provide reliable range images in direct sunlight. Photogrammetry is used to extract 2D data from photographs and reconstructs $3 \mathrm{D}$ objects on the map. This method is flexible, costeffective but has some limitations such as a long processing time and less accuracy compared to laser scanning. Furthermore, it will not work for scenes that have no special features, like a simple plane wall. These authors asserted that laser scanning is suitable for a wide range of measurements at high resolution as it can rapidly and accurately capture the shape of physical objects and represent the scanned scene by a point cloud containing millions of points, each of which has position (XYZ), colour (red/green/blue), and/or intensity data. Compared to other technologies, it is less sensitive to light conditions. However, its utilization is costly and the data collection is time-consuming.

\subsubsection{Below-ground utility detection technologies and methods}

The main problem with below-ground utility detection is that the approximate location of the below-ground utilities has been indicated by marks which are not reliable enough. As the records of buried utility assets contain inaccurate positions and/or depths, some efforts have been made to reduce the amount of incidents caused by these inaccuracies. For instance, the United States created a one-call centre that performs surveys on site and marks the underground utility pipe location on the site, which has reduced the incidents per mile, but the total number of incidents is still high. On the other hand, data acquisition, processing and decision making need to be automated to facilitate measurements and interpretation in real time. Therefore, Huang, Bernd and Bernold [36] developed a new approach at the Construction Automation and Robotics Laboratory, that integrated traditional construction equipment, micro-processing technologies, and "mechatronics" in an innovative way. An electronic device that is capable of real-time warning of utility lines located directly ahead of the digging tool was adapted as an effective mechanism to avoid costly damage and accidents. This device should be attached to the machine and be embedded in its control system. The proposed system differed from traditional metal detection techniques by adopting an active search system. It included (1) a metal detector search coil; (2) a signal processing (control) unit; and (3) a PC equipped with an analogue-to-digital (A/D) converter interface. Therefore, the computer was able to collect real-time magnetic changes data, graph it on screen, sound a signal, and store it in a file for analysis. However, further development is required regarding the programming capability of the microprocessor in order to provide an excellent platform, along with future development of algorithms that are able to engage in real-time pattern 
recognition, and to figure out detailed information about the depth, location, orientation, and even the size of the metal object [36].

There are some technologies, devices and sensors available that are capable of detecting and locating objects below the ground in the vicinity of the excavator on the construction site. These technologies can be used to detect and locate and then map various buried utility networks and prevent utility strikes during excavation. Tanoli et al. [37] classified them in a very similar way to [38] and [39] as follows: 1. Visual (Closed Circuit Television (CCTV)), 2. Electromagnetic and Radio Frequency (low-frequency electro-magnetic field survey, GPR, passive magnetic fields (PMFs)), 3. Acoustics and Vibration (vibro-acoustics, acoustics pipe tracer), 4. Radio Frequency Identification (RFID markers/sensors), 5. Pipe and Cable Locator, 6. Elastic Wave Method, 7. Infrared Thermography, 8. Broadband Electromagnetic/Wave Impedance Probe (WIP), as shown in Fig. 5.

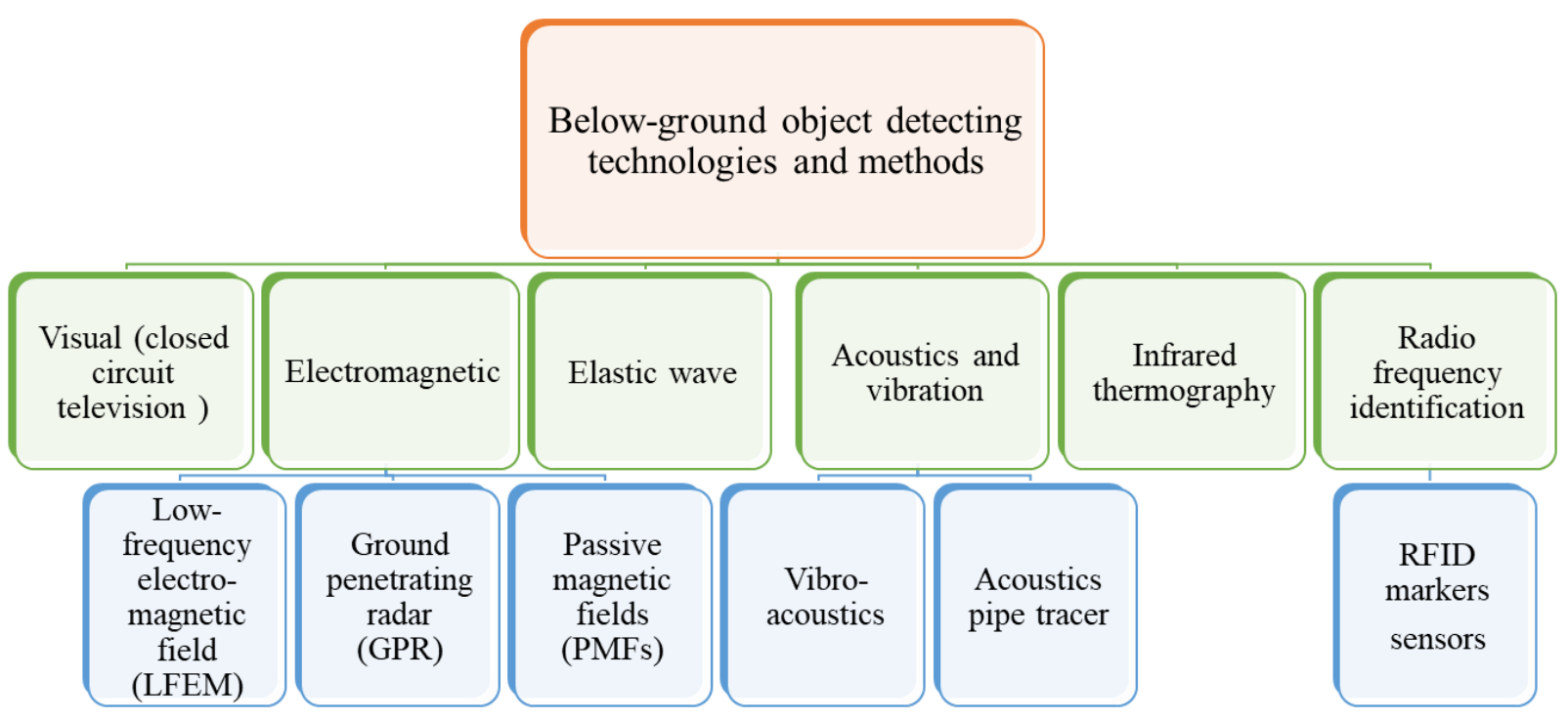

Fig. 5. Hierarchy of technologies and methods for detecting below the ground utility networks during excavation.

Each technology has particular advantages and limitations for the detection and location of buried utilities in different environments. For example, GPR works better on tarmac and should not be used in grass areas as the moisture under the grass reduces the transmission of radar waves, while vibro-acoustics can be utilized for detecting utility networks in grass areas [39]. Another example is PMF, which only detects underground cables with electric current, while LFEM detects both cables and pipes [39]. Li et al. [38] tabulated the limitations associated with each technology in Table 6. However, GPR is the technology that is used in most cases because it detects and maps both metallic and non-metallic utilities at different depths. GPR is an active device that sends electromagnetic waves into the ground and receives the returned signals from buried utilities. The GPR provides a high-resolution utility signature and the investigation depth can be increased, but the image resolution resulting from the investigation reduces significantly. The results at depths of more than $4 \mathrm{~m}$ are not reliable [38][39]. However, it is more difficult for other technologies to detect the depth of the utilities. The GPR properties facilitate the generation of accurate 3D utility maps to facilitate accident prevention during excavation [38].

Table 7. Limitations of underground utility detection technologies, adapted from (Li et al., 2015).

\begin{tabular}{ll}
\hline Technologies & Limitations \\
\hline Magnetometer & - Can only detect ferrous utilities buried shallowly \\
& - Depth estimation is not considered realistic \\
Pipe and cable locator & - Can only detect metallic utilities \\
& - Possible interference from nearby metallic utilities \\
Terrain conductivity & - Unable to detect utilities in high moisture environment \\
method & - Massive amount of data is needed for processing
\end{tabular}


Infrared method

Resistivity measurement

Elastic wave method

Radio frequency

identification

Ground-penetrating radar
- Depth estimation is not considered realistic

- Unable to detect utilities buried deeply

- Not suitable for congested urban environment

- Depth estimation is not considered realistic

- May not be useful as a trace technique

- Data setup and collection is cumbersome

- May not be applicable for mapping paved areas

- Most utilities are too small to be detected

- May be influenced by background noise

- Depth estimation is not possible with acoustic emission

- Attachment of tags to existing utilities is difficult

- Negatively affected by moisture and metallic materials

- Detectability and accuracy are relatively low

- Large signal attenuation in conductive soils

- Highly skilled crews are needed for data interpretation

\subsection{Underpinning methods and technologies}

Ensuring the precise location of various objects and real-time access to data are two critical essentials for reliable accident prevention during excavation [39][10]. These two requirements necessitate higher accuracy and less uncertainty in the objects' pose and state data, as well as system-level application of technology innovations. The accuracy of the monitoring and object detection systems affects the environmental perception uncertainty, which is crucial for the automation and performance improvement of complex environments such as excavation [40]. Environmental noise and outliers affect monitoring and detection system performance in object detection and recognition. For example, the planar segmentation of the truck recognition algorithm can be affected by noisy sensor data, which results in an inaccurate measurement of the truck dimensions and pose [41]. In addition, the sensors in monitoring and object detection systems often measure only one environmental variable, and it is not possible to measure physical effects that do not correspond to the available sensor's measurement principle [40]. So it is difficult to anticipate and prevent accidents based on a single monitoring/ detection system, although integrating data from multiple sensors and monitoring systems, which is called data fusion or a multisensory approach, can enhance the monitoring/detection accuracy and reliability to predict and prevent accidents. Data fusion increases data accuracy by decreasing the associated data uncertainty and boosting a wider spatial and temporal coverage [42].

Further, system-level application of technology innovations is required to continuously monitor and transfer the operation system's safety and performance information, and provide real-time access to data for early detection of abnormality [7]. Besides, the increasing number of automatic data acquisition technologies, such as sensor technologies, has facilitated the development of construction information systems (CISs) and decision support systems (DSSs) [43]. DSSs closely link informatics knowledge, which can improve decision making in all disciplines associated with the built environment, as well as decisions toward higher system safety. The field of construction informatics, also known as construction information technology or communication and information technologies in construction, mainly focuses on the specific issues related to the acquisition, processing, representation/visualization, and interchange of specific construction and operation information. This field is also encouraging the integration and coordination of numerous informatics technologies at a system level. Hence, Hsieh and Hung [44] discussed software components and their roles in the system architecture in a construction information system. They proposed an information system capable of integrating all the monitoring of multiple projects, which facilitates data mining through different projects and different monitoring data. That system was also scalable, meaning it has the capacity to use one platform for multiple large-scale monitoring projects with different attributes, and embraces distributed computing. The information system can also be integrated with other software systems via open standard web services and it can facilitate visualization of the monitoring data [44]. Furthermore, computer science has considered the collision detection concept in different application areas such as computer-aided design and manufacturing, robotics, simulations, and computer games. Some information technology approaches are available which combine computer graphics visualization, geospatial databases, and tracking technology to depict the position of objects proportionate to the equipment operator and the excavation crew [10]. 
It is an essential requirement to integrate monitoring data from multiple sensors in an information management system. In fact, information technology approaches are required to achieve the full potential benefits of data fusion approaches at a system level. The authors believe that excavation systems can significantly benefit from the integration of a multisensory approach and information technology approach. In fact, these are complementary steps toward reliable accident anticipation and prevention during excavation (Fig. 6). Integrating monitoring data from multiple sensors and even multiple projects in an information management system increases the accuracy of the data captured by single monitoring and object detection, as well as the diffusion of that data at a system level. Advanced data fusion algorithms boost both above- and below-ground technologies' accuracy and reliability. Furthermore, advanced communication and information technologies can facilitate accident anticipation and prevention through multi-sensory data visualization, and a real-time warning system. In the following, the technology readiness and development requirements of each approach for safe excavation are discussed in more detail.

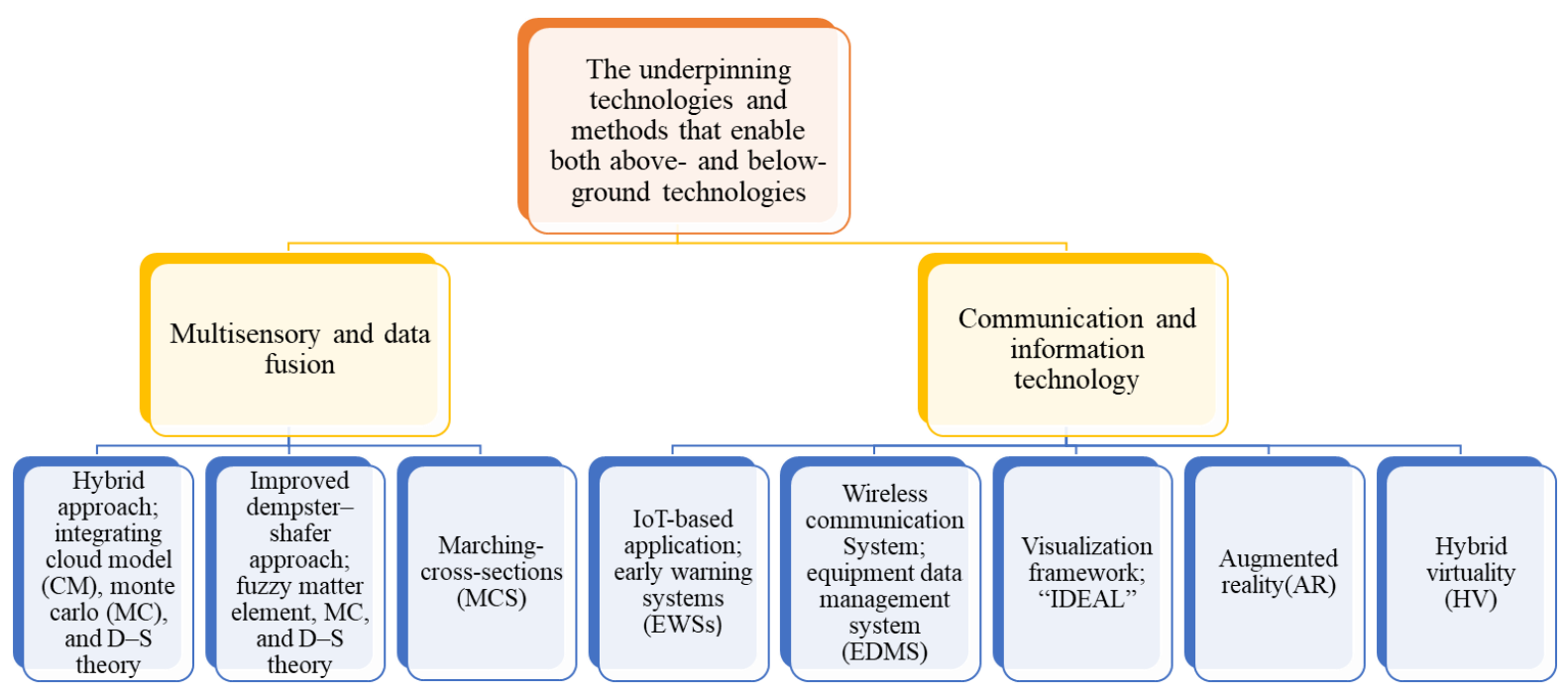

Fig. 6. Hierarchy of general purpose technologies that facilitate system safety.

\subsubsection{Multisensory approach and data fusion for increasing data accuracy and reducing uncertainties}

The different sensors/techniques for above- and below-ground object detection and location have their own limitations. For example, single geophysical techniques are not able to identify all utility types in varying soil conditions [10]. Therefore, the multisensory approach has been developed based on the combined application of geophysical technologies, and if multi-sensor data can be integrated appropriately, more accurate data can be acquired [10][39]. Data fusion is a process in which signals acquired from multiple sensors are integrated based on some algorithms to facilitate decision making by improving the system reliability and reducing the fuzziness of the information [45]. Quantitative and qualitative methods have been used for data fusion such as rough set, the maximum entropy approach, fuzzy integral, and Dempster-Shafer (D-S) evidence theory. The D-S evidence theory is a common and efficient method that overcomes issues such as incomplete data, and accommodates imprecision and uncertainty and any lack of enough prior knowledge [42].

\subsubsection{Multisensory approach for detecting above-ground objects}

Al-Shrouf et al [40] developed a multisensory-based object detection system for monitoring the production process of a bucket-wheel excavator in order to detect large stones to avoid process disturbance. The system developed aimed to be practical and suitable for real-time application with the lowest possible false alarms. Three different physical effects were used to develop a stone detection system. The physical effects were measured by five acceleration sensors, two load cells, and a laser scanner. Each signal was pre-processed, feature-related extracted, and classified. Decision fusion techniques were used to fuse the individual results obtained in order to take a final reliable decision regarding the existence of the object.

Zhang, Wu et al. [42] developed a novel hybrid information fusion approach that integrates cloud model (CM), Monte Carlo (MC), and D-S theory to facilitate safety risk analysis and decision making under uncertainty. They tried to answer a crucial question in complex decision making: "How to synthesize evidence from multiple sources 
that may be conflicting?" (p. 89). Three main stages are incorporated: (1) generation of basic probability assignments (BPAs) using CM; (2) improved D-S rule in evidence combination; and (3) simulation-based decision analysis. The developed approach is capable of synthesizing multi-source information in order to achieve a more accurate result for safety risk perception. Numerous data sources are categorized as hard data (electronic sensors) and soft data (domain experts/humans), and both categories are required for perceiving the safety risk of tunnel-induced building damage or other kinds of infrastructure construction. The results indicate that the uncertainty of the safety risk perception is reduced in a continuous and effective manner.

Zhang, Ding et al. [46] developed an information fusion framework that incorporates 14 influential factors to perceive the risk magnitude of tunnel-induced building damage. They developed an improved Dempster-Shafer approach. It merges the fuzzy matter element (FME), MC simulation technique, and D-S evidence theory to support the construction safety risk perception at an early construction stage. Dempster's rule of combination gives unrealistic results under extreme conflicting items of evidence. Therefore, the authors used Dempster's rule for the conditions where the conflict was less extreme $(\mathrm{K}<0.95)$ and instead used the weighted average rule where the conflict was extreme $(\mathrm{K}>0.95)$.

There is another example of systems which used expert knowledge as soft data. Stentz et al. [47] developed a complex autonomous loading system with over 70 major system components including a hardware processor and software modules, which must all be integrated to make the system able to function successfully. This system aimed to load trucks with soft materials at the speed of human expert operations. It used two scanning laser rangefinders to recognize the truck, measure the soil on the dig face and in the truck, and to detect obstacles in the workspace. The system modifies both its digging and dumping plans based on settlement of the soil as detected by its sensors. This system also applied expert operator knowledge and encoded it into scripts that are adjusted using simple kinematic and dynamics rules to generate very fast machine motions.

\subsubsection{Multisensory approach for detecting below-ground utility}

In addition, there have been some efforts toward more precise data and information acquisition regarding different aspects of utility networks, such as the type, orientation, precise location, etc. These efforts toward greater data accuracy can be categorized into two approaches: 1) sensor evolution, 2) multisensory approach.

- Sensor evolution and detection improvement approach

There have been some accuracy issues with current sensors caused by different factors which need to be improved. For example, according to [48], ground-penetrating radar data acquisition was constrained by equipment availability, weather, legal or logistical constraints, safety or access considerations. Hence the author proposed an approach which is a process to maximize information for the time, cost and hazard risks in which the acquired data will be processed and modelled. A survey was set according to the site condition and was flexible to accommodate unknown factors. In addition to radar data acquisition, calibration, orientation, location and other vital parameters representing the equipment and survey were recorded, and used in the processing and modelling of the data. The final result was a corrected geometric cross-section, interpreting the electrical and magnetic properties of the ground, location, orientation, size and shape of subsurface objects, and the composition of the ground and objects such as the inferred density, porosity, fluid saturation, and other relevant material occurrence properties.

In 2006, [49] introduced a second generation of sensors, which was the evolution of an eddy current sensor based on the induction balance principle to improve its performance. The aim was to identify and locate different types of buried pipelines without excavation. They used modelling to increase the sensitivity of an analogical version of the sensor based on a distributed point source method. A special tag integrating conductive elements was associated with each kind of pipe type. The recognition of the tag allows the identification of the corresponding pipe. They used blind-source-separation algorithms to eliminate the effects of the presence of metallic objects in the area on the reaction of the buried pipes to the signal. Many algorithms can be used to recognize buried tags. Using a wide range of algorithms was necessary to surpass the difficulty of identification and drove them to use an original method of combining the results, seeking to increase the reliability of the final decision [49].

- Multisensory approach

If multi-sensor data can be integrated appropriately, a more accurate and complete buried utility network can be built [10][39]. The North Carolina State University's Construction Automation and Robotics Laboratory (CARL) conducted work to support utility location identification, and safe excavation through working on a multisen sory locating concept [11]. According to CARL, five major data types should be acquired while detecting and locating 
buried objects during excavation: the objects' (1) x-y plane position; (2) z-axis position; (3) shape and size; (4) orientation; and (5) material composition, for example, metal, plastic, concrete, etc. In addition, contractors need to know if the utility is live or not (e.g., power cables). Kolera and Bernold [11] developed an intelligent tool for the excavator operator to "see pipes through the earth". They presented a "fish-finder" type of technology that locates metallic utilities without prior knowledge of their existence, and it was capable of recognizing the direction, depth, size, and centre point of elongated metallic objects. However, their research was limited to metallic object detection, although the computer-controlled actuator was capable of carrying other sensing devices like GPR. Furthermore, they developed a method to display the outcome of the scan as an operator-computer interface, which was able to identify the closest centre-point of a pipe or a cable that crossed the trench area. Dou et al. [39] proposed a "multi-utility multi-sensor" system which used a Marching-Cross-Section (MCS) algorithm for data fusion with five fundamental components, including: utility track initialization, track marching, data association, track updating and track management. The MCS algorithm marches from one scan cross-section to the next by predicting the states of detected utilities in the next cross-section. They discretized the 3D space with scan cross-sections based on automatically generated virtual scan lines or actual survey scan lines. The predicted states of utilities were integrated with hypothesized detections from multiple sensors to obtain better estimations. They concluded that the sensors are either complementary to each other or they verify each other.

3.2.2 Communication, and information technologies to facilitate system-level application of technology innovations

Automatically transferring large amounts of "as-built" construction data (real-time site-level information) in real time depends significantly on advanced wireless communication technologies to collect, communicate and receive electronic data for different applications such as mobility, telematics and telemetry control of construction equipment. Wireless communication integrated with Building Information Modelling (BIM), GPS and the Internet provides the backbone capability necessary for creating intelligent systems, on-site automation and robotics [50]. Robust wireless communication can enhance the applicability of distance measurement technologies during excavation. Accordingly, a model that links BIM with site-level information and data acquisition was developed by [50] to support efficient activity management through real-time communication of data.

As communications within construction management systems were limited to using desktops or laptops, the construction risk management systems described in pre-2014 literature are not applicable on mobile devices. Therefore, Hsiung [51] developed a real-time risk management system called MitiRisk, which is an integration of BIM, mobile web service technology, a desktop, and window mobile phones to improve the efficiency of on-site data acquisition and information sharing of tunnelling projects and deep excavations in urban areas.

More recently, Internet of Things (IoT)-based applications are developing the entire process of monitoring, modelling, and management and also enhancing early warning systems and sustainable decision making [43]. For example, collision prevention in underground construction is highly complex, and its system safety depends on real-time monitoring of various aspects like the status of structures and the environment, as well as human behaviours. Therefore, there is a vital requirement to develop and utilize a framework that integrates the dynamic flow of changing conditions and behaviours to truly achieve an effective early warning system. This requires a seamless integration of physical things and human behaviours to guarantee efficient data collection, analysis and sharing while considering the project performance under time and cost constraints. Accordingly, IoT links a variety of smart objects through (1) real-time monitoring of different objects and networked connection of multiple sensors, (2) reliable transition of heterogeneous information, and (3) intelligent control of things [52].

\subsubsection{Information technology approach for above-ground monitoring and object detection}

IoT-based applications have been utilized for the implementation of early warning systems (EWSs) in structural health monitoring (SHM). Similar systems should be available for earthmoving equipment operation. An EWS merges a wide range of sensors and evaluation methods like signal processing and communication to support accurate forecasting and empower users to advance the level of emergency preparedness, as well as providing intelligent services for users and enterprises, such as scheduling repairs and maintenance in order to lessen the impact of structural degradation [43]. Wang et al. [43] developed two types of warning models based on the relationship between the measured value in real time and a monitored item, established independently, and its standard operating value. In [43] a warning rule is based on changes in the characteristics of a monitored item and its functions. Each type of warning model is divided into three levels, yellow, orange and red, to represent different levels of urgency. A warning signal will be transmitted to the interested party when a given threshold exceeds the established boundaries for a monitored item. The yellow warning level transmits a warning message to the security 
supervisor of the construction project. The orange warning level transmits the security issue to the project manager. A red warning is the highest level of the warning system, when the system identifies a significant security risk that may potentially lead to a serious safety condition, and a hazard notification is transmitted to the supervision department. Additionally, the warning system also records the alarm information and corresponding data stream for later query.

Kim et al. [53] developed a wireless communication system for the remote or autonomous operation of an Intelligent Excavation System (IES), which is an autonomous excavation robot with various hardware and software components including a global environment modeller (GEM) that collects scanning information of the whole operation field, and a local environment modeller (LEM) that models the robot's neighbouring area by the use of a 3D laser scanner. The world modeller (WM) merges the GEM and LEM. Truck/obstacle sensing (TOM) provides obstacle avoidance during loading operations. Attitude and position information of a robotic excavator is acquired via an equipment status sensing (ESS) module. Task plans are created by the excavation task planner (TP), which enables the excavator to move and excavate automatically. The robotic controller creates machine control commands through a control station for movement of the excavator body, and through proportional integral (PI) control for the movement of actuators. This wireless communication system can provide along-range communication required for construction sites, as well as equipment cooperation within the site. A Data Communication Manager (DCM) and an Equipment Data Management System (EDMS) were developed to manage and coordinate large amounts of data and traffic signals (Fig. 7). DCM is a macro-level task planning coordinator, while the EDMS is a micro-level sensing and controlling manager. The DCM and EDMS can coordinate all data and organize the links using a multicast network in order to streamline the communication network. Connection with DCM can definitely reduce the complexity of network modules, as it knows the current status of operation at all times. Bandwidth limitation was considered for DCM establishment. An operator interface of the DCM was developed to notify the operator for the remotely located control station. Figure 6 shows the IES network with and without DCM, which clarifies its importance.

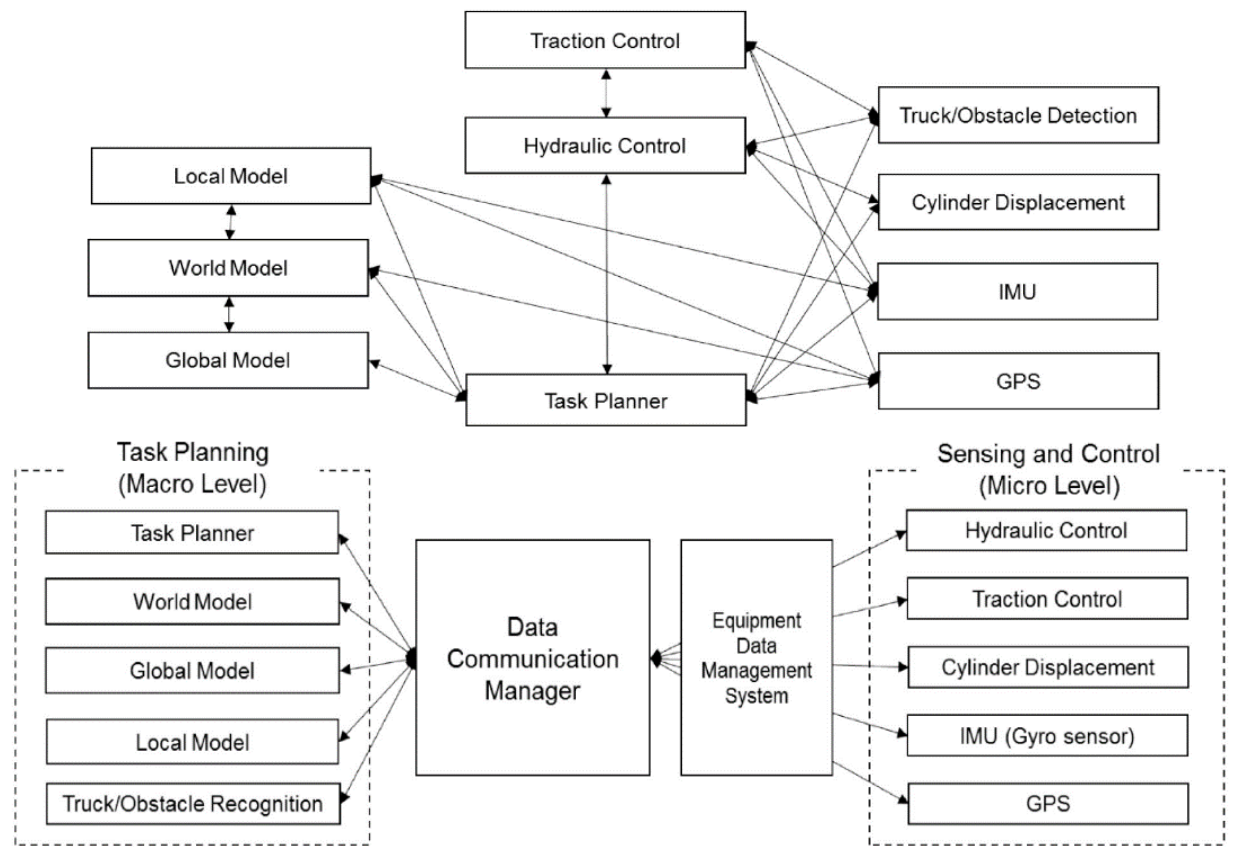

Fig. 7. (a) A chaotic IES network, (b) a coordinated network with DCM. Adapted from [53].

\subsubsection{Information technology approach for below-ground utility detection}

Talmaki and Kamat [10] classified buried utility location techniques in two main categories: (1) a multisensory approach, and (2) an information technology approach (Fig. 8), which are discussed more in detail in the following context. 


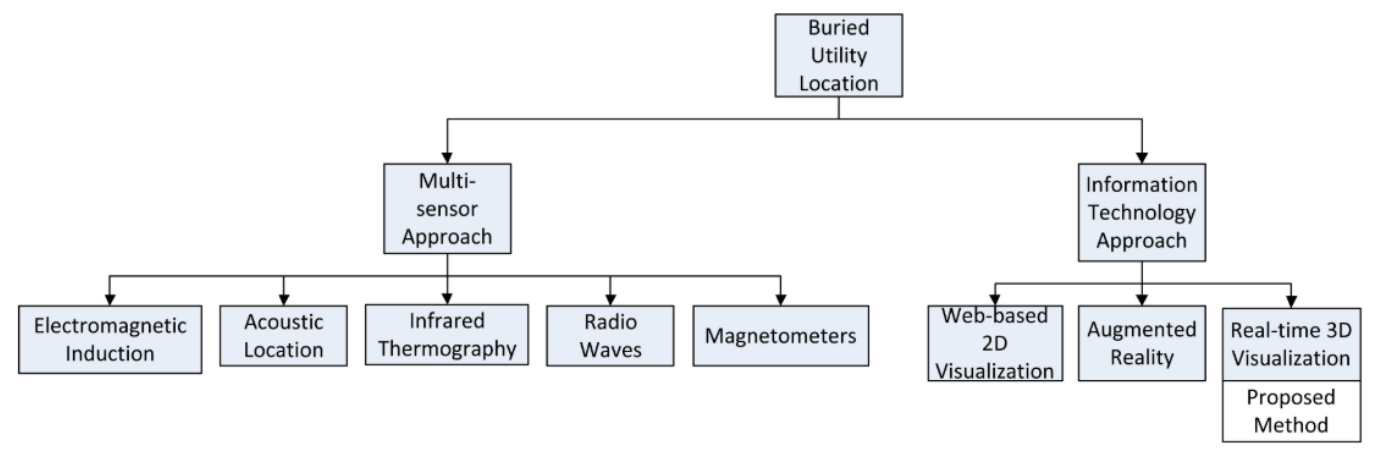

Fig. 8. Buried utilities location and visualization techniques. Adapted from [10].

Information technologies such as web-based services use geospatial databases to store buried utility data and display to the user two-dimensional (2D) or three-dimensional (3D) visualizations on desktop workstations, smart phones, tablets, and personal digital assistants. On the other hand, activity theory is a special approach to look at how humans and computers interact. It explains that computer applications are the extensions of some natural human abilities, and the problem is that technological possibilities have changed the whole nature of the activity [11]. Augmented reality (AR) has been used to visualize below-ground utilities to assist with utility inspection and maintenance, as well as improving the visual perception of buried utilities and excavation safety [54]. By utilizing AR, users receive a mixed view of the real world and virtual below-ground utility lines [10].

However, whether the sensor's data accuracy is improved through a single sensor evolution or by a data fusion approach, there is still a lack of an effective knowledge-based excavation approach to synergize the geospatial utility location and movement of excavation equipment into a real-time 3D spatial context [55][37]. A main issue to execute a knowledge-based excavation approach is the accuracy of the geometric modelling of geospatial utility data [55][37]. Talmaki et al. [55] took key steps toward knowledge-based excavation by describing the computational details of the geometric modelling of utility data for 3D visualization and proximity monitoring. They outlined various stages of a buried utility geospatial data life-cycle, as well as the barriers to using the data for excavation guidance. They developed a visualization framework termed "IDEAL", which is an acronym that illustrates the framework design specifications, including Interactive, Informative, 3-Dimensional, Accurate, and Extensible. The IDEAL framework can demonstrate excavator movement along with 3D visualization of buried utility networks. It can also provide detailed information on the utilities' associated attributes and positional uncertainties that can help the operator in decision making in uncertain circumstances to operate safely and efficiently. For example, visualization of this additional information helps the operator to decide whether to carry on with the heavy equipment or use other non-destructive excavation techniques.

In addition, [56] incorporated uncertainties associated with buried utilities data into a geospatial-AR system for real-time visualization and proximity analysis in a dynamic approach. They presented the uncertainties of buried utilities data as probability bands, described by pairs of band sizes and the probability of the 3D space constructed by buffering at the band size to enclose the true location of utilities [56]. In other research, the Laboratory for Interactive Visualization in Engineering (LIVE) at the University of Michigan developed different AR applications for construction operations planning, inspection, safety, and education, and developed a visual excavator-collision avoidance system [54]. The developed system prevents utility strikes as the excavator operator is able to visualize the data of utilities buried in the vicinity of a digging machine. Furthermore, the hybrid virtuality (HV) term illustrates a scene composed of 3D models that speak for real-world elements such as terrain, surface and underground infrastructure, and on-site equipment which relies on tracking information from sensors instrumented on real-world entities [10]. Talmaki and Kamat [10] identified (1) a lack of accurate position and semantic data on buried utilities, (2) the absence of persistent visual guidance, and (3) a lack of real-time spatial awareness of the proximity of the digging implement to the hidden neighbourhood utilities, as the main safety obstacles facing excavator operators. Therefore, they proposed a hybrid virtuality (HV) method, which is a realtime visual emulation framework to improve equipment operators' knowledge and spatial awareness during excavation. In fact, they developed a computational framework that enables the concurrent visualization of real jobsites using 3D geographic information system models to represent terrain and subsurface utility infrastructure. This framework allows real-time operation monitoring and incorporates proximity sensing and collision detection algorithms, providing audio-visual warnings when safety thresholds are breached. 
However, visualization should be practical and prevent information overload and users should be able to view only the scene that they are concerned with. The user interface should provide essential details without unnecessary information [55][37]. For example, the user should be able to select a specific level of information by turning on/off different layers such as terrain or switch to a utility attributes layer [55].

\subsection{Functionalities to increase adaptive capability}

This section addresses the capabilities driven by the available technology innovations, including accident anticipation, prevention, and response (decision or behaviour) to safety hazard situations such as accidents, as well as the learning potential. It discusses how accidents can be recognized and how to confirm a right decision or behaviour to avoid accidents. Fundamental functions for the accident prevention system showed that accidents can be prevented if there is enough time for situation recognition and enough time for behaviour/decision confirmation [6]. The situation recognition capability is composed of working area estimation, collision risk analysis, then a warning signal should be sent to the people or equipment at risk. Here we present the identified functionalities for accident anticipation, prevention, response and learning that have been developed based on the available technologies. These functionalities highlight the adaptive capability of an excavation system to be resilient against accidents and collisions. They have been categorized and are discussed in three groups: (1) anticipation; situation recognition capability, (2) prevention; decision making capability, and (3) response; behaviour confirmation capability.

\subsubsection{Anticipation: proximity warning}

Research efforts have addressed two different streams to enhance the safety of excavation systems [7]; the first optimized equipment planning and scheduling to reduce the possibility of collisions, in which different activity space requirements are considered to avoid dangerous proximities between different teams of equipment - called the activity workspace. The second applied an automatic monitoring system (advanced sensing and location systems) to reduce the collision risks by warning operators against the potential dangerous proximities in real time. For example, Vahdatikhaki and Hammad [7] developed a system in which the pose, state and speed of equipment are extracted to ensure safe operation within a short time window - called dynamic equipment workspaces (DEW). Location data captured by RTLSs and CV can facilitate the identification of machine-induced safety hazards and the analysis of operation productivity [3].

Accordingly, it is vital to continuously monitor the excavation system and construction site safety and performance for early detection of any abnormality [3][31]. Proximity warning systems (PWS) have been developed to notify workers/equipment that they are coming near objects to prevent collisions [8]. These are based on the objects' location and geometries to identify the spatial relations between objects. There are certain rules on whether to sound an alert or not, after determining the distance between objects. And different sorts of warning signals can be employed for alerting and warning workers and equipment, including visual, acoustic, and tactile signals [57]. The effectiveness of a PWS is based on the sort of technology employed for object tracking. Different technologies can be used, like a global positioning system (GPS), radio frequency (RF) sensing, ultrasonic sensing, vision-based technology, etc. Each technology has particular features such as the detection range, processing time, the existence of additional sensors attached to the object, and installation cost [58][59][57]. Some advantages and disadvantages of the three main types are described below.

RFID can be used for locating objects or object tracking with centimetre-level accuracy. It does not call for highlevel algorithms or computational methods to be utilized on the construction site. It only requires signal strength adjustment and one-step data processing to be implemented on the construction site. It does not depend on lineof-sight and allows object detection even with a barrier in between. Its limitations are that (1) RF signals can encounter interference with multipath fading effects, and (2) objects need to be tagged to be detected [58][59][57]. A GPS sensor identifies the absolute location of objects and returns the latitudinal and longitudinal data of objects, and allows for calculating the distance between the targeted objects. GPS presents location data in a global coordinate system and can cover large areas of construction sites [12]. However, it is not very accurate when the signal can be blocked by other objects or buildings, and it is applicable to open space only. GPSs with a high level of accuracy are available but are relatively expensive [3][57]. And GPS sensors only provide orientation data when the equipment is turning-on-the-spot. This problem can be solved by using two on-board GPS systems or having multiple antennas [12]. The computer vision-based method is very efficient in detecting different objects and returning objects' information such as location, types, velocities, or orientation from construction site images. It does not require supplementary devices such as tags and other sensors to return information. One of the major limitations of the CV-based method is its low accuracy in poor lighting conditions, snow, rain, or dusty weather 
[25][57] [31]. However, one critical requirement for an effective PWS is to provide sufficient and useful information such as the distance between the worker and the equipment, the movement direction of the equipment, or the overall safety level, rather than simply alerting workers or equipment in danger [57]. Considering the abovementioned issues, detailed information from two studies that adopted the computer vision-based approach for hazards detection and communication on construction sites is discussed below.

According to Kim et al. [60], the simplest mechanism to perceive safety issues on construction sites is to assess the context of every single entity (worker, or equipment) on site. For example, several hazards can disturb workers' safety, and such circumstances can affect each other in respect of the degree of danger. For instance, a congested area is more unsafe for a worker than a spacious area when an excavator is approaching that area, because in a congested area the worker has less awareness of the surroundings and less chance of avoiding the excavator. Kim et al. [60] asserted that using a single safety level that integrates various probable hazards at a certain time into one single value is a more accurate and precise approach to assess safety. In addition, this integrated safety level must be communicated in a useful and informative manner to facilitate environmental perception on time and take instant action. In addition, [60] developed an on-site safety assessment system for construction sites to quantify a worker's current safety level by evaluating the safety level of every single entity. This system contained two key functional modules, a vision-processing module and a safety assessment module. In the vision-processing module, spatial information on all entities on the site was acquired by a digital camera that took construction site images. Then, fuzzy inference was used to evaluate the safety levels of each entity (workers or equipment) from the spatial information. The spatial safety information was based on proximity and crowdedness, and struck-by accidents were adopted as a safety hazard for validation. Fuzzy logic was developed by following a human's reasoning process pattern. So this can be a replacement for the safety supervisor's judgment of the jobsite safety conditions. Subsequently, safety levels can be automatically originated without human intervention.

Kim et al. [57] developed a vision-based hazard avoidance system to proactively notify workers of possible unsafe situations. The system consists of three modules: (1) a vision-based module for site monitoring that discovers site hazards using an image capture device and wearable devices, (2) a safety assessment module to assess the safety level of each object based on fuzzy-based reasoning, and (3) a visualization module that creates useful information like hazard orientation, distance, and safety level. The system helps workers to proactively prevent accidents by exposing the augmented reality of hazard information via the interface of a wearable device. The information covers the worker's safety level, distance from the risky equipment, and the equipment orientation. Safety level and distance details are calculated when the worker is pinpointed in global perspective images. For calculating the equipment orientation, the present direction of the worker should be considered, and is obtained from the wearable device. A field experiment to test the system proved its efficacy. This system helps both managers and workers to perceive a hazardous situation and respond to it in a timely manner [57]. Many more proximity zone systems have been developed to warn either workers or equipment. For example, [8] developed and tested a methodology to design hazard zones around construction equipment. They described hazard zones as areas that pedestrian workers should stay away from during construction operations. Their method is applicable in site planning and construction site workers' safety education. The methodology has six steps: "(1) equipment footprint; (2) initial safety boundary; (3) equipment function; (4) operation reaction time; (5) equipment braking distance; and (6) creation of the hazard zone" (p. 5016005). They generated hazard zones for three pieces of construction equipment: dump truck, backhoe loader, and excavator. The results can be used to boost awareness of dangers from neighbouring construction equipment for construction site personnel. They also developed a userfriendly hazard zone creation tool and database for safety managers [8]. Jo et al. [59] also developed a proximity detection and control system for the prevention of collision accidents caused by excavators. This system utilized RFID and an Around View Monitor (AVM) to detect the workers' path and alert them to dangerous situations through an alarm device. Also, a control system was designed to shut down the excavator automatically when required. The RFID technology was used to detect real-time hazard proximity and an AVM system was employed to add visual information to support the operator in distinguishing workers from other objects. Many automobile companies have developed AVM systems as driver-assistance systems that provide the operator with a $360^{\circ}$ image of the surroundings [59].

As concerns anticipating utility strikes during excavation, various technologies such as GPR are used to detect and map both metallic and non-metallic utilities at distinct depths. GPR can produce depth information for 2D GIS buried utility records to create accurate 3D utility maps. The integration of GPR and GPS provides high accuracy in 3D location of buried utilities [38]. Precise 3D geospatial utility data is essential to prevent utility 
strikes during excavation [38]. Tanoli et al. [37] proposed a method for avoiding underground utility strikes during excavation using GPR to map the underground utility networks in an excavation site. The acquired data was used for 3D modelling in the machine guidance systems. An uncertainty band was added to the utility position to increase the safety level. The developed system could increase the excavator operator's awareness regarding the existence of utilities in the vicinity of the excavation area to facilitate accident prevention.

\subsubsection{Prevention: accident-free navigation}

A navigation system with an efficient and safe path-planning algorithm is essential for the safety of automated excavation systems. Equipment needs to discover a path to move safely from a start point to a destination without a collision. A path-planning method that deals with environmental conditions and constraints and achieves completeness, efficiency, and optimality is a critical fundamental for developing an automated excavation equipment [61]. Completeness indicates the intelligence to determine the existence or non-existence of a path solution without undertaking an endless loop. Efficiency means the speed of finding a certain path solution. Optimality is the ability to discover the most efficient path based on required criteria such as the moving distance, and energy consumption. Kim et al. [61] suggested intelligent navigation strategies to enhance the efficiency of excavation execution for automated earthwork systems, which include global navigation and local navigation strategies based on a scanned environment on a computer. The global navigation strategy establishes mobile paths according to the size of buffers for the equipment's safety. It suggests a method to arrive at the destination while avoiding mobile obstacles. The local navigation strategy produces efficient paths, which minimize equipment movement by breaking down a working area into several small zones, and recommends the order of movement between zones and the movement patterns within each zone.

In general, equipment-related collision risks can be efficiently mitigated through integration of the planning and monitoring safety phases. However, Vahdatikhaki and Hammad [7] argued that there is still an approach - a middle-level approach - that has not been fully investigated. In fact, the initial path planning can lose its efficiency in the face of unforeseen situations that may occur during a project. On the other hand, a real-time warning is not able to provide the required time window for path re-planning. At the monitoring stage, it is essential to have a solution that is able to accurately foresee the equipment operation for a long-enough duration of time to allow the equipment to re-adjust their initial planned paths to prevent accidents in near real-time. Earlier, the DEW approach was discussed, based on the continuous tracking of a wide range of information such as equipment pose, state, and speed. This approach relies on the equipment stoppage time to calculate the required space reservation for providing an accident-free domain in the instant future. But this approach is only useful to trigger warnings or immediately stop the equipment, and does not provide a predictive capability for equipment to foresee possible accidents and execute path re-planning to avoid them. Accordingly, [7] developed another approach called LookAhead Equipment Workspaces (LAEWs) to establish a comparatively expanded spatial risk assessment of the space around the equipment (for the next $10 \mathrm{~s}-1 \mathrm{~min}$ ) and to take account of the proximity-based risks and the site visibility conditions relating to the future states of the equipment. In fact, this approach inspects the equipment operation pattern, the visibility status surrounding the equipment, and the required information for creating DEWs. Equipment risk maps can be generated from the results of these analyses and they illustrate the risk distribution around the equipment. These risk maps can be deployed to produce the LAEWs related with a definite risk level. The LAEWs ensure that different pieces of the equipment's initial path are collision-free, or they will correct their path planning to stay away from potential collision. Figure 9 shows LAEWtruck and LAEWexcavator for different risk levels. If the risk level declines, the LAEW expands in size. The LAEWtruck helps the truck to investigate possible collisions in the initial path, and re-plan the path if required. And the excavator should apply LAEWexcavator to ensure a safe path and avoid collision with the truck or other equipment. In addition, as the excavator is usually digging ground, the space analysis for the excavator can be enlarged and encompass space below ground level. Then LAEW can be applied for underground utilities strike avoidance. This method is based on a simulation model and it will be continuously updated by the data collected during operation. The workspace shape can be correlated with the potential future poses and states of the equipment by leveraging the cyclic pattern of equipment activity as well as their movement characteristics information [7]. 


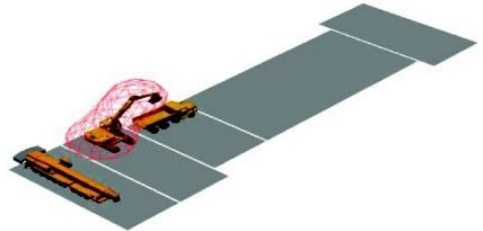

(a)

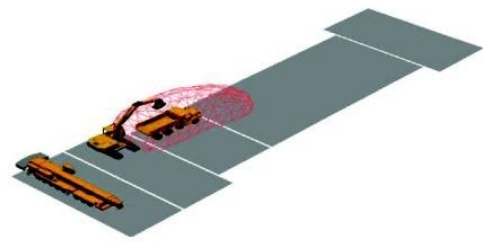

(c)

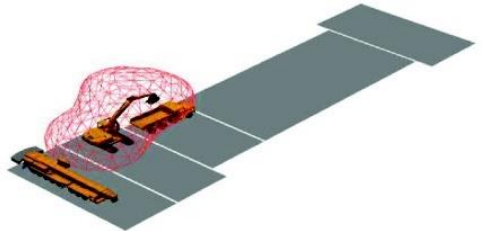

(b)

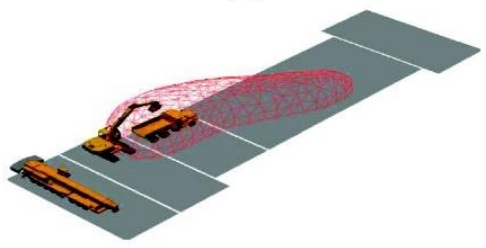

(d)

Fig. 9. (a) LAEWtruck with higher risk level, (b) LAEWtruck with lower risk level, (c) LAEWexcavator with higher risk level, (d) LAEWexcavator with lower risk level. Adapted from [7].

\subsubsection{Response: knowledge-based adaptive control system}

Seward et al. [12] introduced the concept of an autonomous system having "values" by means of utility theory. Seward et al.'s [12] focal point was carrying out safe navigation of the excavator platform on a construction site. According to this paper, the operational safety assurance for the robot increases with upgrading of the mobility and autonomy. Mobile robots need to have adaptation capabilities to maintain safety while operating in dynamic and unstructured environments. This brings various challenges in developing a safe autonomous system, as the system cannot be restricted to one approach and different behaviours should be set up to maintain safety. The main aim of [12] was to develop a strategy, and a method of implementation, that will let an autonomous vehicle - an excavator - move from one point to a selected target point with a behaviour that is aligned with its "values". The simulation results showed that intelligent systems can be originated with a changing behaviour capability that features one of two "safety-conscious" and "task-achieving" personalities.

It is very difficult to design and implement a fully automated system that produces efficient and safe earthwork plans autonomously [61]. Lancaster University has been involved in the development of an autonomous robot excavator called LUCIE (the Lancaster University Computerized Intelligent Excavator) [7]. One of the main aims of the project was to add autonomy to the system, with a capability to deal with obstructions, enhancing system safety. A prototype was developed that achieved this capability. They designed a highly modular system that included the possibility of adding underdeveloped technologies such as a hidden service pipe detection system and navigation system in the future. One of the innovative parts of this project was that Seward and Garman [7] introduced the concept of a Safety Manager (SM), which was investigated earlier by a project called Safe-SAM. SM was developed because a robotic excavator requires a knowledge-based adaptive control system to deal with an unstructured and unpredictable environment. Safety Manager necessarily needs a separate processor and should be cognizant of objects in the working environment, and only authorize safe operation. As a metaphor, in biological terms, it performs like the 'conscience' of the system. Another example is a nuclear protection system, which is not concentrated on the functional process of the plant, but prevents hazards arising[7].

Later on, Seward et al. [1] introduced a safety module to LUCIE's automation model to perform system safety analysis. The main challenge in the design stage was to ensure that the machine is able to perform its basic tasks in a zero-accident-prone environment. System safety analysis should consider different factors regarding the interaction with an unstructured construction site, including: environmental state establishment during operation, the location of obstacles, the location of the machine itself, taking action in response to environmental changes, moving to a different location, etc. The LUCIE hardware system utilized different sensors, such as potentiometers on the joints for angle measurement, an axis tilt sensor, an optical laser distance sensor for obstacle detection, satellite GPS for location and navigation, and had three processors: an activities manager to send high-level commands for navigation and digging, a low-level controller for the arm and tracks, and the SM to oversee the environment and ensure safe operation. The SM was an autonomous processor that was essentially responsible for all forms of safety hazards. In fact, a safety executive software was developed in which safety analysis is spread over the whole system [1]. The safety manager was developed based on "the generation and maintenance of safe excavator operational states. Two general safe states were defined: an internal safe state and an external safe state, both of them had to be attained for safe system operation. The internal safe state identified the internal 
system status, such as communication requirements between processors and the mechanical and sensory operation requirements for safety integrity. The external safe state identified the environmental safety aspects, including environmental conditions for which safety integrity was achievable" [1] (p. 36). Their system safety analysis included three main processes: hazard analysis, determining basic functionality, and defining hazard.

Hazard analysis provides a comprehensive and early understanding of the required SM functionality [1]. The safety analysis centre was a hazard analysis, to ensure that the outcome of the analysis designates the requirements specification for the whole system. The hazard analysis aimed to identify which parts of LUCIE's functionality increase hazard, what underlying functionality can be adoptedto prevent the hazard, or what extra functionality might be added. Therefore, definition of the underlying functionality is an essential stage, but this is not the same as a requirements specification. The functional definition must assist the hazard analysis process and not the requirements definition. On the other hand, a hazard definition must necessarily deal with a whole class of real situations, and the rationale of dangerous situations must include the state of a complex unstructured environment. Therefore, to prevent any assumptions being made about the system and environment and their interactions, and to ensure that no hazardous situations were omitted from the analysis, hazards were defined in the most comprehensive possible way, which included three main classes:

- Collision with an object on the surface

- Collision with an underground object

- Toppling of the excavator.

In other work, [12] implemented Real-time Control System-Reference Model Architecture (RCS-RMA) in LUCIE. RCS-RMA is a framework for developing complex robotic systems, and Partially Observable Markov Decision Processes (POMDP) are proposed for managing probabilistic decision making at appropriate hierarchical levels of RCS which can deal with uncertainties. Accordingly, the system provides "defence in depth" against accidents and it provides a strong basis for safety management within the action selection/decision making processes. An upper RCS level provides strategic safety planning while a lower level facilitates safe tactical decisions. Actually, the system was developed to evaluate the robot's behaviour when it needs to avoid a trench avoiding the risk of a toppling accident - under diverse operating conditions. It depends on knowledge of the terrain conditions available to the robot. Therefore, the robot will be able to select a safe path towards the target.

\subsubsection{Learning: AI and VR}

Monitoring technologies provide the opportunity to learn from what went wrong in the system [63], as well as learning from what went right that led to accident avoidance and prevention. One of the efforts toward developing $\mathrm{AI}$ is to integrate vision-based technologies with deep learning (machine learning) methods [29], which provides the means to subdivide images, and recognize patterns that can be used for potential learning to facilitate autonomous decision making. Therefore, an action can be executed through utilizing algorithms that are able to use learning in various contexts. For example, the accident situations caused by equipment in different environments can be automatically identified, and autonomously avoided. In addition, VR provides the opportunity to assess the effect of different actions in the environment [34] and use it for learning potential.

\section{Discussion}

Numerous technologies and methods have been developed to ensure the safety of humans, equipment, and properties on the excavation site. To fully benefit from the advances in this area, it is very important to be aware of all the different kinds of available technologies and methods that have been developed for this purpose, and to classify them in different categories associated with their application and role in excavation system safety. All the identified technologies and methods have been categorized in three main groups to highlight their impacts and roles in excavation system safety. The first group represents the available monitoring and detection technologies that can detect different objects such as equipment, humans, utility networks, etc. The excavation system usually contains two distinct environments with different characteristics, namely above-ground and below-ground. Therefore, authors have clearly categorized and defined the different sets of technologies that are required to detect objects above or below the ground.

On the other hand, a resilient system should be reliable and robust, which means that it must function accurately and effectively in any situation, even in unpredicted situations. It is very important to consider the reliability and effective functioning of monitoring and object detection systems to be able to ensure their positive impact on excavation system safety. If such systems are not sufficiently reliable, and cannot accurately detect potential collisions or effectively ensure awareness of a possible hazard on time, they cannot support efficient real-time 
decision making to enhance the safety and resilience of the excavation system. Therefore, the second group of technologies introduces essential enablers for reliable and effective monitoring and object detection systems, including: 1) the application of multisensory and data fusion approaches, in order to increase the data accuracy and reduce uncertainties and fuzziness of data captured by a single sensor, and 2) system-level application of technology innovations which can be facilitated through data storage and communication technologies and methods. The first one increases the reliability of safety decisions that have been made based on the captured data, and the second one ensures the effective establishment of data and decisions in the system through general purpose technologies and methods to store, communicate and process the data captured by the different types of technologies. In addition, the third group represents the technical functionalities developed that increase the adaptive capability of the excavation system. This adaptive capability can be enhanced through accident anticipation, prevention, the response to safety hazards during excavation, and learning from it. The association and relation between those functionalities and different aspects of an adaptive resilient system have been studied in subsection 3.3. So far, the development of these functionalities has included proximity warning, safe navigation, below-ground utility detection, knowledge-based adaptive control systems, and machine learning for AI and advanced safety of excavation systems. However, there are still certain shortcomings in the current research that need to be addressed in order to achieve the full potential provided by technologies to enhance excavation system safety.

The readiness level of different technologies and the identified limitations of current technologies and research, as well as future directions for research are discussed below.

\subsection{Reliable prevention strategies for immediate decision making}

The authors were curious to determine if researchers have taken cognizance of the effects of technology innovations on system safety or taken a resilience engineering perspective in developing and testing new technologies. The system safety concept, which is identical with resilience engineering, was manifested while studying the intersection of the safety and resilience research areas. In the existing literature there is substantial evidence regarding the rationale of resilience in the arena of safety and the incorporation of resilience into safety programs. The increasing complexity of excavation sites due to the automation of equipment and processes is forcing researchers and practitioners to consider the advantages of going beyond conventional safety management and taking more steps toward resilient excavation systems. Resilience engineering necessitates accurate near realtime situation recognition, decision making and behaviour confirmation capabilities for excavation equipment. This calls for a continuous systematic analysis of machine-environment interaction in near real-time, to enable a reliable accident anticipation capability in the excavation system. Ultimately, an excavator should be able to manage its interaction with unstructured and unpredictable environments during task execution, whilst achieving its operational goals and enhancing system safety. For automated and autonomous excavators, an accurate realtime environmental perception is the key for enhancing system safety during operation. The study showed that, in general, location data captured by RTLSs can facilitate the identification of machine-induced safety hazards [3]. In addition, real-time access to objects' pose and state data, and ensuring the precise location of utility pipelines are critical factors for the anticipation and prevention of accidents in excavation systems [39][10]. One example is the work of Chae and Yoshida [6], who used RFID technology to develop a system for accident prevention of workers with heavy equipment.

\subsubsection{Trade-off strategies}

Correspondingly, appropriate accident prevention strategies should be adopted immediately after accident anticipation, to facilitate adaptive behaviour of the equipment. If accidents occur, not only does the excavation system lose personnel, property or equipment, but the operation process can be disturbed on various scales, and consequently the whole system's performance and productivity will be affected negatively. The literature reviewed clearly discussed the need to avoid accidents not only for the sake of safety, but to maintain a desired level of productivity of the system. At the same time, the resilience engineering approach puts significant emphasis on developing appropriate accident prevention strategies to promote both safety and productivity. This can lead to securing both system safety and productivity, which means that equipment, personnel, utility networks, and any other element of the system can function safely, with zero or minimal negative effect on the system's productivity. Accordingly, one of the main capabilities of an autonomous excavator should be the incorporation of an automatic process of making safe decisions that evaluates different endogenous and exogenous risks and acts accordingly to reduce/mitigate the identified risks without human intervention. Some researchers developed a knowledge-based adaptive control system - the Safety Manager - in order to achieve the abovementioned goal 
and this was a principal step toward applying an autonomous accident prevention capability. However, the safety manager modules developed used a very simple rule for decision making that does not consider the trade-offs between system safety and system productivity and performance. Thus, more realistic and robust strategic planning needs to be accommodated within the existing 'safety manager' modules to overcome the problems of real complex excavation systems. There is a crucial need for further research exploring efficient decision making algorithms that can remove the need for managerial/human level intervention during excavation. Reliable prevention strategies should be developed for immediate decision making in emergency and emergent events, rather than simple reactions such as stopping the operation (stopping the machine), and waiting for managerial decision making, which results in reduced productivity. This calls for efficient strategies that deal with trade-offs between performance, safety and productivity.

\subsubsection{Utility strike prevention strategies}

The existing safety manager modules only developed accident prevention strategies for avoiding collisions with above-ground objects. There is a significant lack of efficient strategies to prevent excavator collision with belowground utility networks.

\subsubsection{Automatic generation of prevention strategies}

Fundamental functions for the accident prevention system showed that accidents can be prevented if there is enough time for situation recognition and behaviour/decision confirmation. This necessitates continuous monitoring of a wide range of vital signals that can be provided recently by wearable technologies to provide early warning systems in the case of unsafe conditions and behaviour [9]. In addition, more recently, IoT is providing a shift from reactive safety management methods to automated proactive methods aligned with specific excavation site characteristics [52]. As an example, Ding et al. [52] developed an IoT-based safety early warning system with real-time sensing, transmitting, perceiving and controlling layers, which enabled emergency reactions by all workers who were on the underground construction site. The system focused on the real-time integration of safety monitoring and warning systems to identify and prevent potential accidents. This system reduced the reaction time to an emergent situation, which provides a greater probability of saving lives and assets [52]. However, there is still a significant lack of appropriate methods, frameworks or models that can automatically generate appropriate accident prevention strategies for different situations and scenarios. The existing literature has discussed numerous methods and innovative technologies for anticipating and detecting potential accidents during excavation, but determining how to avoid those accidents effectively or how to autonomously respond to accident-prone situations is still a big burden on those who work in the area of excavation system safety. Agent-based modelling is highly required to simulate and test different accident prevention strategies for excavation systems.

\subsection{Integration of technologies}

Different studies have been conducted to develop and test different technology innovations that monitor, detect, locate, and track different objects at excavation sites. However, an excavator has a greater risk of accidents than other earthmoving equipment due to its articulated shape, and it is challenging to monitor an object with a large degree of freedom and to estimate the risk that its three-dimensional extent poses on excavation sites. Even integrating different technologies such as a time-of-flight camera with a charge-coupled camera could not overcome this issue, as the camera's maximum operating range was 7.5 metres, insufficient for an excavation site [31]. In addition, an autonomous earthmoving equipment system should provide full sensor coverage to estimate the terrain topology, compute optimized digging trajectories and consider potential hazards at the construction site. Typically, an autonomous excavation system requires a broad set of sensors to sense the digging face, recognize and locate the truck, and detect objects in the workspace, which includes above-ground and underground objects, and avoid collisions with those objects while achieving its desired performance. Therefore, the monitoring and detection of objects during excavation is far more complex than other earthmoving operations. In addition, as a variety of objects are involved in the excavation system, there is no single technology innovation available that is capable of enhancing excavation system safety all by itself. Therefore, various monitoring and object detection technologies should be integrated to acquire, process and transfer data on the whole excavation system comprehensively. The application and integration of various technologies might be a solution that takes forward the deficiencies embedded in any particular technology. This requirement also illustrates the need for advanced data fusion algorithms for monitoring complex excavation systems. Some researchers developed data fusion algorithms for combining different technologies for either above-ground object detection or underground utility network detection. For example, one study fused CV-based and RTLSs methods for detecting objects above the ground, and another study fused data from five different sensors to detect utility networks under the ground. But 
underground utility detection is still facing a huge uncertainty as concerns precise location of utility lines, their material type, etc. Thus, both sections still require further research and development. Data fusion is a very well developed approach for many other construction projects. For example, Zhang et al. [46] developed an information fusion framework that incorporates 14 influential factors to perceive the risk magnitude of tunnel-induced building damage. They developed an improved Dempster-Shafer approach that merges the fuzzy matter element (FME), MC simulation technique, and D-S evidence theory to support the perception of construction safety risk at an early construction stage. Dempster's rule of combination gives unrealistic results under extreme conflicting items of evidence. Therefore, the authors used Dempster's rule for the conditions where the conflict was less extreme $(\mathrm{K}<0.95)$ but used the weighted average rule where the conflict was extreme $(\mathrm{K}>0.95)$. This kind of study should be adopted for increasing the accuracy of object detection in excavation systems.

\subsection{System-level application of technologies}

Most of the developed technologies are focused on the location or tracking of a single object such as an earthmover, or only provide partial information on the environment. But a system-level application of technologies is required to address the complex interaction of the various objects in the excavation system.

\subsection{Accuracy level of technologies}

In addition, some uncertainty and inaccuracy exist in the pose and state data acquired from real-time location systems (RTLSs) for detecting or tracking objects above the ground, as well as in the precise location of buried pipelines and cables. These inaccuracies and uncertainties increase the probability of accidents and strikes, which leads to huge maintenance costs and lower productivity rates. An excavator (or excavator operator) needs to have precise information on the pose (position and orientation) and state of objects over the ground, as well as the horizontal and vertical location of utility lines, which is a major limitation of current practice [10]. Some researchers even tried to visualize the uncertainty of the acquired utility data [38][56]. This area of research needs further development to reach a level sufficient to ensure the avoidance of utility strikes during excavation. On the other hand, most of the available technologies are able to acquire and transfer object pose data, but acquiring and transferring the state of objects (idle, busy, loading material, unloading material, etc.) has been difficult and less accurate. Nevertheless, the evaluation of the effect of technology innovations on excavation system safety can be carried out more precisely through studying the accuracy level of every single available technology and their system-level application, which is beyond the scope of this paper.

\subsection{Reliable communication technologies}

Since fast and accurate data exchange and connectedness capabilities are required to communicate unsafe situations in real time, general purpose technologies such as $5 \mathrm{G}$ data communication are essential to achieve the desired level of safety, provided that security and safety factors such as cyber-security are fully considered in their development and application. Nowadays, large-scale automated monitoring systems can be easily and economically deployed based on various wireless communications and wireless sensor network technologies to monitor construction sites. The acquired information can also be used as safety measurement indicators, facilitating life cycle safety management [44], and can be used for potential learning. In addition to safety improvements, the monitoring system can provide real system response information that is different from computer simulations. The information acquired during operation monitoring can be transferred to a computer store and a machine learning approach can turn this into knowledge, and facilitate learning from experience that will lead to more robust system design and management. This information has three important applications: (1) it provides decision support systems (DSS) to act against catastrophic events, (2) it can enhance computer simulation by providing feedback to the models, and (3) data mining creates valuable knowledge of engineering system design [44].

\subsection{Machine learning approaches}

Among the different technologies, the computer vision-based method has received significant attention as it has the potential to solve many of the current issues regarding system-level monitoring, object state identification, etc. However, the recent research trend raises new challenges to cover the pose tracking of articulated equipment for reliable performance of computer vision-based methods on real construction sites [31]. In addition, object recognition in construction site digital images is challenging due to the changing viewpoints, illumination, occlusions, and scale. However, despite the existence of several advanced algorithms for the application of computer vision-based methods for construction projects, the machine learning approach has not been adequately 
utilized for enhancing the safety of excavation systems. For instance, instead of objects, it should be capable of recognizing actions resulting from articulated motions based on the video captured from a fixed camera [3].

\subsection{Simulation requirements}

In a complex engineering system, such as an excavation system that encompasses huge uncertainties, overseeing different scenarios that reflect uncertainty and unexpectedness can prepare the system for responding effectively to safety and/or productivity disturbances. Thus the simulation of different scenarios containing embedded uncertainties in different technology innovations, as well as uncertainties embedded in the excavation environment, is highly recommended. Furthermore, IoT provides an opportunity for the communication and connectedness of different objects in the excavation system. The possibility of real-time communication and information exchange among different objects at the excavation site has significantly increased. Reliable decisions for system safety can be made through different communication and information exchange protocols such as a centralized or decentralized approach or a hybrid communication approach. Examining the effectiveness of these approaches through computer simulations is highly desired.

\subsection{Technology roadmap development}

And finally, since there is a broad range of technology innovations with different capabilities, the authors suggest developing a technology roadmap for connected autonomous excavation. This is highly desirable for different construction bodies including construction companies, commercial firms, manufacturers, standards bodies, researchers and others. It would help to identify specific technology classes and requirements for further research, development and investment toward an autonomous connected excavator that accurately perceives the operation environment and has adaptive capabilities to react appropriately to safety hazards. The ultimate level of automation in a connected autonomous plant roadmap may be to achieve a fully automated excavator that has the autonomy to make optimized decisions for system safety without human intervention.

\section{Conclusion}

This paper investigated the available technology innovations that can enhance excavation system safety by conducting a systematic literature review. In general, this study tried to assess if monitoring technology developments can enhance the resilience of excavation sites systematically, rather than having a small effect on the safety management of the system. A real-time environmental perception facilitated by automatic monitoring and object detection systems can be used to provide effective accident prevention strategies. This is a very important requirement for enhancing excavation system safety. The identified technologies, categorized and discussed in three main groups, include (1) monitoring and object detection technologies, (2) underpinning methods and technologies, and (3) functionalities for accident anticipation, prevention, response, and learning. Categorizing these technologies and methods in different groups clarified the vital role of each group in the overall system safety, as well as the different technology level readiness of each group. Therefore, the research gaps and areas for further research and advances to achieve safer and resilient excavation systems could be underlined in each group. The discussion section presents all the identified research gaps and further research development recommendations that will be useful for technology developers, researchers, and policy makers.

To enhance system safety, the excavation system should have anticipation, prevention, response and learning capabilities to not only anticipate and prevent accidents, but also respond to unsafe and accident-prone situations, and learn from them. This is a fundamental requirement to enable the excavation system to deal with the complexity and uncertainty inherent in its dynamic environment if it intends to promote safety while keeping productivity in mind. Proximity warning, below-ground utility detection, safe navigation, knowledge-based adaptive control systems, and learning are capabilities generated from available technology innovations to anticipate, prevent and respond to accidents and learn from them. The results showed the potential positive impacts of technology innovations on excavation system safety through implementing the abovementioned capabilities.

\section{Acknowledgements}

I hereby thank University College London, Department of Civil, Environmental and Geomatic Engineering for funding my $\mathrm{PhD}$ study, and providing the opportunity for me to conduct this study. I also highly appreciate Professor Liz Varga and Dr Yukun Hu for their excellent supervision and support during my PhD study. 
[1] D. Seward, C. Pace, R. Morrey, and I. Sommerville, "Safety analysis of autonomous excavator functionality," Reliab. Eng. Syst. Saf., vol. 70, no. 1, pp. 29-39, 2000.

[2] R. Francis and B. Bekera, "A metric and frameworks for resilience analysis of engineered and infrastructure systems,” Reliability Engineering and System Safety, vol. 121. pp. 90-103, 2014.

[3] F. Vahdatikhaki, A. Hammad, and H. Siddiqui, "Optimization-based excavator pose estimation using real-time location systems,” Autom. Constr., vol. 56, pp. 76-92, 2015.

[4] M. M. Soltani, Z. Zhu, and A. Hammad, "Skeleton estimation of excavator by detecting its parts," Autom. Constr., vol. 82, no. July, pp. 1-15, 2017.

[5] F. Vahdatikhaki, A. Hammad, and H. Siddiqui, "Optimization-based excavator pose estimation using real-time location systems," Autom. Constr., vol. 56, pp. 76-92, 2015.

[6] S. Chae and T. Yoshida, "Application of RFID technology to prevention of collision accident with heavy equipment,” Autom. Constr., vol. 19, no. 3, pp. 368-374, 2010.

[7] F. Vahdatikhaki and A. Hammad, "Risk-based look-ahead workspace generation for earthwork equipment using near real-time simulation," Autom. Constr., vol. 58, pp. 207-220, 2015.

[8] X. Shen, E. Marks, N. Pradhananga, and T. Cheng, "Hazardous Proximity Zone Design for Heavy Construction Excavation Equipment.," J. Constr. Eng. Manag., vol. 142, no. 6, pp. 5016001-5016006, Jun. 2016.

[9] I. Awolusi, E. Marks, and M. Hallowell, "Wearable technology for personalized construction safety monitoring and trending: Review of applicable devices," Autom. Constr., vol. 85, no. July 2016, pp. 96 $106,2018$.

[10] S. Talmaki and V. R. Kamat, "Real-time hybrid virtuality for prevention of excavation related utility strikes,” J. Comput. Civ. Eng., vol. 28, no. 3, 2014.

[11] B. T. Kolera and L. E. Bernold, "Intelligent Utility Locating Tool for Excavators.," J. Constr. Eng. Manag., vol. 132, no. 9, pp. 919-927, Sep. 2006.

[12] D. Seward, C. Pace, and R. Agate, "Safe and effective navigation of autonomous robots in hazardous environments," Auton. Robots, vol. 22, no. 3, pp. 223-242, 2007.

[13] J. Bergström, R. Van Winsen, and E. Henriqson, "On the rationale of resilience in the domain of safety: A literature review," Reliability Engineering and System Safety, vol. 141. pp. 131-141, 2015.

[14] L. J. Bellamy, M. Chambon, and V. van Guldener, "Getting resilience into safety programs using simple tools - a research background and practical implementation,” Reliab. Eng. Syst. Saf., vol. 172, pp. 171$184,2018$.

[15] A. Carmeli, Y. Friedman, and A. Tishler, "Cultivating a resilient top management team: The importance of relational connections and strategic decision comprehensiveness,” Saf. Sci., vol. 51, no. 1, pp. 148$159,2013$.

[16] M. F. Costella, T. A. Saurin, and L. B. de Macedo Guimarães, "A method for assessing health and safety management systems from the resilience engineering perspective," Saf. Sci., vol. 47, no. 8, pp. 1056-1067, 2009.

[17] D. Tranfield, D. Denyer, and P. Smart, "Towards a Methodology for Developing Evidence-Informed Management Knowledge by Means of Systematic Review," British Journal of Management, vol. 14, no. 3. pp. 207-222, 2003.

[18] F. Habib, M. Bastl, and C. Pilbeam, "Strategic responses to power dominance in buyer-supplier relationships: A weaker actor's perspective,” Int. J. Phys. Distrib. Logist. Manag., vol. 45, pp. 182-203, 2015.

[19] Q. N. Hong and P. Pluye, "A Conceptual Framework for Critical Appraisal in Systematic Mixed Studies Reviews,” J. Mix. Methods Res., vol. 13, no. 4, pp. 446-460, 2019.

[20] L. Lusiantoro, N. Yates, C. Mena, and L. Varga, "A refined framework of information sharing in perishable product supply chains,” Int. J. Phys. Distrib. Logist. Manag., vol. 48, no. 3, pp. 254-283, 2018 . 
[21] F. Vandatikhaki, S. M. Langari, A. Taher, K. El Ammari, and A. Hammad, "Enhancing coordination and safety of earthwork equipment operations using Multi-Agent System," Autom. Constr., vol. 81, pp. 267-285, Sep. 2017.

[22] E. R. Azar and B. McCabe, "Part based model and spatial-temporal reasoning to recognize hydraulic excavators in construction images and videos," Autom. Constr., vol. 24, pp. 194-202, Jul. 2012.

[23] E. Viljamaa and I. Peltomaa, "Intensified construction process control using information integration," Autom. Constr., vol. 39, pp. 126-133, 2014.

[24] H. Tajeen and Z. Zhu, "Image dataset development for measuring construction equipment recognition performance,” Autom. Constr., vol. 48, pp. 1-10, 2014.

[25] W. Fang, L. Ding, B. Zhong, P. E. D. Love, and H. Luo, “Automated detection of workers and heavy equipment on construction sites: A convolutional neural network approach," Adv. Eng. Informatics, vol. 37, pp. 139-149, 2018.

[26] H. Kim, H. Kim, Y. W. Hong, and H. Byun, "Detecting Construction Equipment Using a Region-Based Fully Convolutional Network and Transfer Learning,” J. Comput. Civ. Eng., vol. 32, no. 2, 2018.

[27] E. R. Azar, S. Dickinson, and B. McCabe, "Server-Customer Interaction Tracker: Computer VisionBased System to Estimate Dirt-Loading Cycles," J. Constr. Eng. Manag., vol. 139, no. 7, pp. 785-794, Jul. 2013.

[28] E. Rezazadeh Azar and B. McCabe, "Automated Visual Recognition of Dump Trucks in Construction Videos,” J. Comput. Civ. Eng., vol. 26, no. 6, pp. 769-781, 2011.

[29] W. Fang et al., "Computer vision applications in construction safety assurance," Autom. Constr., vol. 110, no. November 2019, p. 103013, 2020.

[30] J. Seo, S. Han, S. Lee, and H. Kim, "Computer vision techniques for construction safety and health monitoring," Adv. Eng. Informatics, vol. 29, no. 2, pp. 239-251, 2015.

[31] M. M. Soltani, Z. Zhu, and A. Hammad, "Framework for Location Data Fusion and Pose Estimation of Excavators Using Stereo Vision,” J. Comput. Civ. Eng., vol. 32, no. 6, 2018.

[32] J.-J. Chen, W. Zhang, and J.-H. Wang, "Data Fusion Analysis Method for Assessment on Safety Monitoring Results of Deep Excavations,” J. Aerosp. Eng., vol. 30, no. 2, p. B4015005, 2017.

[33] V. Gikas, "Three-dimensional laser scanning for geometry documentation and construction management of highway tunnels during excavation," Sensors (Switzerland), vol. 12, no. 8, pp. 11249-11270, 2012.

[34] M. Golparvar-Fard, J. Bohn, J. Teizer, S. Savarese, and F. Peña-Mora, "Evaluation of image-based modeling and laser scanning accuracy for emerging automated performance monitoring techniques," Autom. Constr., vol. 20, no. 8, pp. 1143-1155, 2011.

[35] E. D. Marks, T. Cheng, and J. Teizer, "Laser scanning for safe equipment design that increases operator visibility by measuring blind spots," J. Constr. Eng. Manag., vol. 139, no. 8, pp. 1006-1014, 2013.

[36] X. Huang, D. Bernd, and L. E. Bernold, "Innovative Technology Development for Safe Excavation.," J. Constr. Eng. Manag., vol. 122, no. 1, p. 91, Mar. 1996.

[37] W. A. Tanoli, A. Sharafat, J. Park, and J. W. Seo, "Damage Prevention for underground utilities using machine guidance,” Autom. Constr., vol. 107, no. September 2018, p. 102893, 2019.

[38] S. Li, H. Cai, and V. R. Kamat, "Uncertainty-aware geospatial system for mapping and visualizing underground utilities," Autom. Constr., vol. 53, pp. 105-119, 2015.

[39] Q. Dou et al., "3D buried utility location using a marching-cross-section algorithm for multi-sensor data fusion,” Sensors (Switzerland), vol. 16, no. 11, 2016.

[40] L. Al-Shrouf, N. Szczepanski, and D. Soeffker, "Online feature-based multisensor object detection system for bucket-wheel excavators," Int. J. Adv. Manuf. Technol., vol. 82, no. 5-8, pp. 1213-1226, Feb. 2016

[41] A. Stentz, J. Bares, S. Singh, and P. Rowe, "Robotic excavator for autonomous truck loading," Auton. Robots, vol. 7, no. 2, pp. 175-186, 1999.

[42] L. Zhang, X. Wu, H. Zhu, and S. M. AbouRizk, "Perceiving safety risk of buildings adjacent to tunneling excavation: An information fusion approach," Autom. Constr., vol. 73, pp. 88-101, 2017. 
[43] J. Wang, Y. Fu, and X. Yang, "An integrated system for building structural health monitoring and early warning based on an Internet of things approach," Int. J. Distrib. Sens. Networks, vol. 13, no. 1, 2017.

[44] Y.-M. Hsieh and Y.-C. Hung, “A scalable IT infrastructure for automated monitoring systems based on the distributed computing technique using simple object access protocol Web-services," Autom. Constr., vol. 18, no. 4, pp. 424-433, Jul. 2009.

[45] Y. F. Hou, Q. R. Meng, M. M. Tong, and T. Liang, "Study on gas monitoring technology based on information fusion,” J. Coal Sci. Eng., vol. 16, no. 1, pp. 57-63, 2010.

[46] L. Zhang, L. Ding, X. Wu, and M. J. Skibniewski, “An improved Dempster-Shafer approach to construction safety risk perception,” Knowledge-Based Syst., vol. 132, pp. 30-46, 2017.

[47] J. Ryde and N. Hillier, "Performance of Laser and Radar Ranging Devices in Adverse Environmental Conditions," J. F. Robot., vol. 26, no. 9, pp. 712-727, Sep. 2009.

[48] G. R. Olhoeft, "Maximizing the information return from ground penetrating radar," J. Appl. Geophys., vol. 43, no. 2-4, pp. 175-187, Mar. 2000.

[49] A. Zitouni, L. Beheim, R. Huez, and F. Belloir, "Smart electromagnetic sensor for buried conductive targets identification,” IEEE Sens. J., vol. 6, no. 6, pp. 1580-1590, 2006.

[50] P. R. Zekavat, S. Moon, and L. E. Bernold, "Securing a wireless site network to create a BIM-allied work-front," Int. J. Adv. Robot. Syst., vol. 11, no. 1, pp. 1-15, 2014.

[51] B.-C. B. Hsiung, "A novel mobile information system for risk management of adjacent buildings in urban underground construction,” Geotech. Eng., vol. 45, no. 3, pp. 52-63, 2014.

[52] L. Y. Ding et al., "Real-time safety early warning system for cross passage construction in Yangtze Riverbed Metro Tunnel based on the internet of things," Autom. Constr., vol. 36, pp. 25-37, Dec. 2013.

[53] J. Kim, S. S. Lee, J. Seo, and V. R. Kamat, “ChaoticDa,” Autom. Constr., vol. 90, no. February, pp. 166-177, 2018.

[54] A. H. Behzadan, S. Dong, and V. R. Kamat, "Augmented reality visualization: A review of civil infrastructure system applications," Adv. Eng. Informatics, vol. 29, no. 2, pp. 252-267, 2015.

[55] S. Talmaki, V. R. Kamat, and H. Cai, "Geometric modeling of geospatial data for visualization-assisted excavation,” Adv. Eng. Informatics, vol. 27, no. 2, pp. 283-298, 2013.

[56] X. Su, S. Talmaki, H. Cai, and V. R. Kamat, "Uncertainty-aware visualization and proximity monitoring in urban excavation: a geospatial augmented reality approach,” Vis. Eng., vol. 1, no. 1, 2013.

[57] K. Kim, H. Kim, and H. Kim, "Image-based construction hazard avoidance system using augmented reality in wearable device," Autom. Constr., vol. 83, no. April, pp. 390-403, 2017.

[58] S. Chae and T. Yoshida, "Application of RFID technology to prevention of collision accident with heavy equipment,” Autom. Constr., vol. 19, no. 3, pp. 368-374, 2010.

[59] B.-W. Jo, Y.-S. Lee, J.-H. Kim, D.-K. Kim, and P.-H. Choi, "Proximity warning and excavator control system for prevention of collision accidents," Sustain., vol. 9, no. 8, 2017.

[60] H. Kim, K. Kim, and H. Kim, "Vision-based object-centric safety assessment using fuzzy inference: Monitoring struck-by accidents with moving objects," J. Comput. Civ. Eng., vol. 30, no. 4, pp. 1-13, 2016.

[61] S. K. Kim, J. Seo, and J. S. Russell, "Intelligent navigation strategies for an automated earthwork system,” Autom. Constr., vol. 21, no. 1, pp. 132-147, 2012.

[62] D. W. Seward and A. Garman, "The sofeware development for an intelligent robot," Comput. Control Eng. J., vol. 7, no. 2, pp. 86-92, 1996.

[63] E. Hollnagel, Safety-II in practice: developing the resilience potentials. Taylor \& Francis., 2017. 